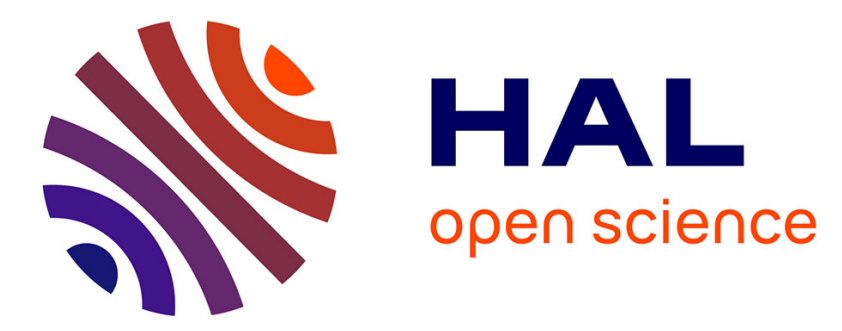

\title{
Shape optimization methods for the Inverse Obstacle Problem with generalized impedance boundary conditions
}

\author{
Fabien Caubet, Marc Dambrine, Djalil Kateb
}

\section{- To cite this version:}

Fabien Caubet, Marc Dambrine, Djalil Kateb. Shape optimization methods for the Inverse Obstacle Problem with generalized impedance boundary conditions. Inverse Problems, 2013, 29 (11). hal00780735

\section{HAL Id: hal-00780735 \\ https://hal.science/hal-00780735}

Submitted on 24 Jan 2013

HAL is a multi-disciplinary open access archive for the deposit and dissemination of scientific research documents, whether they are published or not. The documents may come from teaching and research institutions in France or abroad, or from public or private research centers.
L'archive ouverte pluridisciplinaire HAL, est destinée au dépôt et à la diffusion de documents scientifiques de niveau recherche, publiés ou non, émanant des établissements d'enseignement et de recherche français ou étrangers, des laboratoires publics ou privés. 


\title{
Shape optimization methods for the Inverse Obstacle Problem with generalized impedance boundary conditions
}

\author{
Fabien Caubet*, Marc Dambrine ${ }^{\dagger}$, Djalil Kateb*
}

January 24, 2013

\begin{abstract}
We aim to reconstruct an inclusion $\omega$ immersed in a perfect fluid flowing in a larger bounded domain $\Omega$ via boundary measurements on $\partial \Omega$. The obstacle $\omega$ is assumed to have a thin layer and is then modeled using generalized boundary conditions (precisely Ventcel boundary conditions). We first obtain an identifiability result (i.e. the uniqueness of the solution of the inverse problem) for annular configurations through explicit computations. Then, this inverse problem of reconstructing $\omega$ is studied thanks to the tools of shape optimization by minimizing a least squares type cost functional. We prove the existence of the shape derivatives with respect to the domain $\omega$ and characterize the gradient of this cost functional in order to make a numerical resolution. We also characterize the shape Hessian and prove that this inverse obstacle problem is unstable in the following sense: the functional is degenerated for highly oscillating perturbations. Finally, we present some numerical simulations in order to confirm and extend our theoretical results.
\end{abstract}

Keywords: geometric inverse problem, shape calculus, generalized impedance boundary conditions, Ventcel boundary conditions, identifiability result

AMS Classification: 49Q10, 49Q12, 34A55, 35Q93

\section{Contents}

$\begin{array}{llr}1 & \text { Introduction } & 2\end{array}$

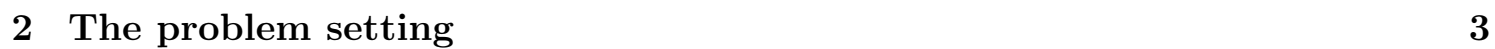

2.1 General notations and reminders . . . . . . . . . . . . . . . . . . . . . . . . 3

2.2 The studied problem . . . . . . . . . . . . . . . . . . . . . . . . . . 4

\begin{tabular}{|lr}
3 & On the identifiability result on rings \\
\hline
\end{tabular}

4 Existence of shape derivatives $\quad 8$

$4.1 \quad$ Characterization of $v_{\theta}$. . . . . . . . . . . . . . . . . . . . . . . . . . . . . 9 9

4.2 Differentiability of $\boldsymbol{\theta} \mapsto v_{\theta}$. . . . . . . . . . . . . . . . . . . . . . . . . . . . 10

4.3 Existence of shape derivatives . . . . . . . . . . . . . . . . . . . . . . . . . . 10

${ }^{*}$ LMAC, EA 2222, Université de Technologie de Compiègne, F-60200 Compiègne, France

${ }^{\dagger}$ IPRA, FR 2952, Université de Pau et des Pays de l'Adour, CNRS, F-64013 Pau, France 
$\begin{array}{llr}5 & \text { Shape calculus } & \mathbf{1 1}\end{array}$

5.1 Shape derivative of the problem . . . . . . . . . . . . . . . . . . . 12

5.2 Shape derivatives of the functional . . . . . . . . . . . . . . . . 15

$5.2 .1 \quad$ First order shape derivatives of the functional . . . . . . . . . . . . 15

5.2 .2 Second order shape derivatives of the functional . . . . . . . . . . 16

5.3 Instability of the problem $\ldots \ldots \ldots \ldots \ldots$

$\begin{array}{lll}6 & \text { Numerical experiments } & 18\end{array}$

6.1 Framework for the numerical simulations . . . . . . . . . . . . . . . . . . 18

6.2 Reconstruction of some obstacles . . . . . . . . . . . . . . . . . . . . . . 19

6.3 Influence of some parameters $\ldots \ldots \ldots \ldots$. . . . . . . . . . . 20

\begin{tabular}{lll}
\hline 7 & Conclusion & 21
\end{tabular}

\begin{tabular}{|l|l|}
\hline A Some results concerning Ventcel boundary conditions & 21
\end{tabular}

\begin{tabular}{ll}
\hline B Shape differential calculus & 23
\end{tabular}

B.1 Tangential differentiation . . . . . . . . . . . . . . . . . . . . . . 24

B.2 Shape derivative of functionals $\ldots \ldots \ldots \ldots \ldots . \ldots \ldots$

B.3 Shape tangential derivation . . . . . . . . . . . . . . . . . . . . 24

\section{Introduction}

A method to solve the inverse problem of reconstructing an object living in a larger domain is the shape optimization methods and particularly the geometric shape optimization. This kind of studies was realized for the impedance electric tomography by Afraites et al. in [2] or for the Stokes and Navier-Stokes equations by Badra et al. in [6] or Caubet et al. in 14, 13, 12 using technics of shape differentiation with respect to the domain. These technics are exposed for example in the books of Henrot et al. and Sokołowski et al. [21, 27]. In the previous references [2, 6, 14, 13], the authors proved that the studied inverse problems, treated as a shape optimization problems, are severely ill-posed (i.e. unstable), for both Dirichlet and Neumann boundary conditions on the object. Thus, they have to use some regularization methods to solve them numerically.

Here, we focus on the case of generalized impedance boundary conditions (of order two) on the obstacle, precisely Ventcel boundary conditions which deal with a Laplace-Beltrami operator. This kind of non classical boundary conditions appear in the modeling of thin layer or of corrosion effects. The study of this type of boundary conditions is an emerging research theme and the analysis of the Ventcel boundary conditions was recently made by Bonnaillie-Noël et al. in [7]. We can here mentioned the work of Haddar et al. in [18] where they propose a new construction of generalized impedance boundary conditions and develop a complete mathematical analysis for the approximate problem. The construction of generalized impedance boundary conditions is also treated for example by Antoine et al. in [4], by Poignard in [26] or by Haddar et al. in [19]. One topic is then to identify the coefficients corresponding to these conditions as treated by Bourgeois et al. in [9, 8]. Another is to identify the shape while the coefficients are known. We consider this question here: our aim is to adapt the classical shape optimization methods used to solve inverse problems to the case of immersed obstacles modeled by Ventcel boundary conditions. In the recent work [11, Cakoni et al. address the question of recovering simultaneously the unknown boundary and the unknown coefficients by an integral equation approach. 
A method to solve this inverse problem of determining the shape of an immersed obstacle is then to make a boundary measurement on the exterior boundary. A first study of this problem is given by Cakoni et al. in [10] using a boundary integral equation method in the spirit of the method proposed by Kress et al. in [22]. In particular, in [10], the authors give an example of non identifiability result. Even if we do not obtain a general identifiability result (as the one stated for Robin boundary conditions by Bacchelli in [5]), we here prove it for annular configurations with the same center using two well chosen measurements. Then, following previous works on electric impedance topography (see [17, 2, 3]) or on the same topic in the Stokes and Navier-Stokes cases (see [6, 14, 13]), our strategy is to minimize a least squares functional.

We prove the existence of shape derivatives for this type of boundary value problem pursuing the studies initiated by Dambrine et al. in [15]. We compute the shape gradient and characterize the shape Hessian. We then prove, using a local regularity argument, that the Riesz operator corresponding to this shape Hessian is compact and it follows that the functional is degenerated for highly oscillating perturbations. Finally, we make some numerical simulations in order to confirm and complete our theoretical study. These numerical experiments point out that, contrary to the case of classical boundary conditions treated in [2, 14, we can achieve acceptable reconstruction without regularization methods. Indeed, the presence of the Laplace-Beltrami operator in the generalized impedance boundary conditions of order two seems to somehow regularize the problem.

This paper is organized as follows. We first introduce the precise problem under consideration and useful results. Then, we prove an identifiability result in the case of an annulus in dimension two. In Section 4 , we prove the existence of shape derivatives (of order one and two) when Ventcel boundary conditions are imposed. The differentiation with respect to the domain of the problem and of the cost functional are detailed in Section 5. This section ends with the proof of the instability of the inverse problem. Finally, we make some numerical experiments which underline the fact that we do not need some regularization method to obtain efficient results. In order to be complete, we recall some results concerning the Ventcel boundary conditions in Appendix A and some reminders of useful shape differential calculus in Appendix B

\section{The problem setting}

\subsection{General notations and reminders}

Let us introduce the notations that we adopt in this paper. We denote by $\mathrm{L}^{p}, \mathrm{~W}^{m, p}$ and $\mathrm{H}^{s}$ the usual Lebesgue and Sobolev spaces. For $k \in \mathbb{N}$ and an open set $\Omega \subset \mathbb{R}^{d}$ $(d \geq 2)$, we denote by $C_{c}^{k}(\Omega)$ the space of functions with continuous $k$ first derivatives compactly supported in $\Omega$ and by $\|\cdot\|_{k, \infty}$ its natural norm. We note in bold the vectorial functions and spaces: $\mathbf{L}^{p}, \mathbf{W}^{m, p}, \mathbf{H}^{s}$, etc. Moreover, $\mathbf{n}$ represents the external unit normal to $\partial \Omega$, and for a smooth enough function $u$, we note respectively $\partial_{\mathrm{n}} u$ and $\partial_{\mathrm{nn}}^{2} u$ the normal derivative and the second normal derivative of $u$. We also denote by $\mathcal{M}_{d, d}$ the space of the matrix of size $d \times d$. The tangential differential operators will be noted by the subscript $\tau$. We recall some definitions and useful results in Appendix B. For more details on tangential differential operators, we refer for example to Section 5.4.3 in [21]. In particular, we recall the following integration by parts result which will be used many times in this paper.

Theorem 2.1. Let $\Omega$ be a bounded open set of $\mathbb{R}^{d}(d \geq 2)$ with a $C^{2}$ boundary. Let 
$f \in \mathrm{W}^{1,1}(\partial \Omega)$ and $\boldsymbol{W} \in \mathbf{W}^{1,1}(\partial \Omega)$. Then,

$$
\int_{\partial \Omega} \boldsymbol{W} \cdot \nabla_{\tau} f=\int_{\partial \Omega}-f \operatorname{div}_{\tau} \boldsymbol{W}+\mathrm{H} f \boldsymbol{W} \cdot \mathbf{n}
$$

where $\mathrm{H}$ is the mean curvature of $\partial \Omega$.

\subsection{The studied problem}

Let $\Omega$ be a smooth (at least $C^{3}$ ) bounded open set of $\mathbb{R}^{d}$, where $d \geq 2$ is an integer representing the dimension. Let $\delta>0$ be a fixed (small) real number. We define

$$
\begin{array}{r}
\mathcal{O}_{\delta}:=\left\{\omega \subset \subset \Omega \text { be a smooth (at least } C^{3} \text { ) open set such that } \mathrm{d}(x, \partial \Omega)>\delta \forall x \in \omega\right. \\
\text { and such that } \Omega \backslash \bar{\omega} \text { is connected }\} .
\end{array}
$$

Let $g \in \mathrm{H}^{3 / 2}(\partial \Omega)$ be an admissible boundary measurement and let $f \in \mathrm{H}^{5 / 2}(\partial \Omega)$ such that $f \neq 0$. We then consider, for $\omega \in \mathcal{O}_{\delta}$, the following overdetermined boundary values problem:

$$
\left\{\begin{aligned}
-\Delta u & =0 & & \text { in } \Omega \backslash \bar{\omega} \\
u & =f & & \text { on } \partial \Omega \\
\partial_{\mathrm{n}} u+\alpha u+\beta \Delta_{\tau} u & =0 & & \text { on } \partial \omega \\
\partial_{\mathrm{n}} u & =g & & \text { on } \partial \Omega,
\end{aligned}\right.
$$

where $\alpha>0$ and $\beta<0$ are fixed real number.

We assume here that there exists $\omega^{*} \in \mathcal{O}_{\delta}$ such that $(2.2)$ has a solution. This means that the measurement $g$ is perfect, that is to say without error. Thus, we consider the following geometric inverse problem:

$$
\text { find } \omega \in \mathcal{O}_{\delta} \text { and } u \text { which satisfy the overdetermined system 2.2. }
$$

To solve this inverse problem, we consider, for $\omega \in \mathcal{O}_{\delta}$, the least squares functional

$$
J(\omega):=\frac{1}{2} \int_{\partial \Omega}\left|\partial_{\mathrm{n}} u(\omega)-g\right|^{2},
$$

where $u(\omega) \in \mathrm{H}^{1}(\Omega \backslash \bar{\omega})$ solves

$$
\left\{\begin{aligned}
-\Delta u & =0 & & \text { in } \Omega \backslash \bar{\omega} \\
u & =f & & \text { on } \partial \Omega \\
\partial_{\mathrm{n}} u+\alpha u+\beta \Delta_{\tau} u & =0 & & \text { on } \partial \omega,
\end{aligned}\right.
$$

measuring in the misfit to data in the $\mathrm{L}^{2}$ sense. Notice that, according to Theorem A.2. the boundary value problem $(2.5)$ admits a unique solution $u \in \mathrm{H}^{3}(\Omega \backslash \bar{\omega})$.

Then, we try to minimize the least squares criterion $J$ :

$$
\omega^{*} \in \underset{\omega \in \mathcal{O}_{\delta}}{\operatorname{argmin}} J(\omega) .
$$

Indeed, if $\omega^{*}$ solves the inverse problem (2.3), then $J\left(\omega^{*}\right)=0$ and (2.6) holds. Conversely, if $\omega^{*}$ solves the optimization problem (2.6) with $J\left(\omega^{*}\right)=0$, then it is a solution of (2.3).

Remark 2.2. Using the local regularity of the solutions in a neighborhood of $\partial \omega$, notice that we can only assume that $\partial \Omega$ is Lipschitz and that $f \in \mathrm{H}^{1 / 2}(\partial \Omega)$ and $g \in \mathrm{H}^{-1 / 2}(\partial \Omega)$ (see for example [6, 13, 14]). However, in order to simplify the proof and the notations, we assume the announced regularity.

Moreover, we could assume that the measurement $g$ is made only on a part $O \subset \partial \Omega$ and not on the whole exterior boundary as made for example in [6, 13]. 
The needed functional tools. Let us introduce the following notation for $k \in \mathbb{N}$ :

$$
\mathrm{H}_{\partial \Omega}^{k}(\Omega \backslash \bar{\omega}):=\left\{u \in \mathrm{H}^{k}(\Omega \backslash \bar{\omega}), u=0 \text { on } \partial \Omega\right\} .
$$

Let us define $\Omega_{\delta}$ an open set with a $C^{\infty}$ boundary such that

$$
\{x \in \Omega ; \mathrm{d}(x, \partial \Omega)>\delta / 2\} \subset \Omega_{\delta} \subset\{x \in \Omega ; \mathrm{d}(x, \partial \Omega)>\delta / 3\} .
$$

Then, in order to make a shape sensitivity analysis (of order two), we define

$$
\boldsymbol{U}:=\left\{\boldsymbol{\theta} \in \mathbf{W}^{3, \infty}\left(\mathbb{R}^{d}\right) ; \operatorname{supp}(\boldsymbol{\theta}) \subset \overline{\Omega_{\delta}}\right\} \quad \text { and } \quad \boldsymbol{U}:=\left\{\boldsymbol{\theta} \in \boldsymbol{U} ;\|\boldsymbol{\theta}\|_{3, \infty}<\min \left(\frac{\delta}{3}, 1\right)\right\}
$$

as the space of admissible deformations. These spaces permit to perturb only the object $\omega$ and not the fixed domain $\Omega$. Notice that if $\boldsymbol{\theta} \in \mathcal{U}$, then $(\mathbf{I}+\boldsymbol{\theta})$ is a diffeomorphism. For such a $\boldsymbol{\theta} \in \mathcal{U}$ and $\omega \in \mathcal{O}_{\delta}$, we check that $\Omega=(\mathbf{I}+\boldsymbol{\theta})(\Omega)$ and we define the perturbed domain

$$
\omega_{\theta}:=(\mathbf{I}+\boldsymbol{\theta})(\omega)
$$

which is so that $\omega_{\theta} \in \mathcal{O}_{\delta}$.

Let $T>0$, that we will have to fix small. We use the shape calculus introduced by Murat and Simon in [25]. Thus, we consider the function

$$
\phi: t \in[0, T) \mapsto \mathbf{I}+t \boldsymbol{V} \in \mathbf{W}^{3, \infty}\left(\mathbb{R}^{d}\right),
$$

where $\boldsymbol{V} \in \boldsymbol{U}$. Note that for small $t, \phi(t)$ is a diffeomorphism of $\mathbb{R}^{d}$ and that $\phi^{\prime}(0)=\boldsymbol{V}$ vanishes on $\partial \Omega$ and even on the tubular neighborhood $\Omega \backslash \overline{\Omega_{\delta}}$ of $\partial \Omega$. For $t \in[0, T)$, we define

$$
\omega_{t}:=\phi(t)(\omega)
$$

where $\phi$ is defined by (2.8). For the rest of the paper, we use a subscript " $t$ " to indicate that the quantity is defined on the time $t$ dependent domain. For instance, $\mathbf{n}_{t}$ is the external unit normal of $\Omega \backslash \overline{\omega_{t}}$.

Finally, we introduce the following notation: for $\boldsymbol{V} \in \boldsymbol{U}$,

$$
V_{\mathrm{n}}:=\boldsymbol{V} \cdot \mathbf{n}
$$

\section{On the identifiability result on rings}

In this section, we will analyze the question of identifiability of the shape $\omega$. The question is the following: does a measurement (or several measurements) determine uniquely the domain $\omega$ ? This kind of result was recently proved by Bacchelli in [5] for Robin boundary conditions. In the case of generalized impedance boundary condition, the literature is reduced to the discussion by Cakoni et al. in [11].

In order to try to answer to this question concerning the non classical Ventcel boundary conditions, let us focus on particular geometries in the bi-dimensional case. More precisely, we consider an annulus with two concentric circles: the inner circle $\Gamma_{i}:=\partial \omega=\rho S^{1}$ has a radius $\rho$ and we denote by $R$ the radius of the outer circle $\Gamma:=\partial \Omega=R S^{1}$ (where $S^{1}$ denotes the unit sphere). Hence,

$$
\Omega \backslash \bar{\omega}:=\left\{x \in \mathbb{R}^{2}, \rho<|x|<R\right\}=B_{R} \backslash \overline{B_{\rho}} .
$$


Let $u$ the solution of the following problem

$$
\left\{\begin{array}{rlll}
\Delta u & =0 & \text { in } \Omega \backslash \bar{\omega}=B_{R} \backslash \overline{B_{\rho}} \\
u & =f & \text { on } \Gamma=\partial \Omega=R S^{1} \\
\partial_{\mathrm{n}} u+\alpha u+\beta \Delta_{\tau} u & =0 & \text { on } \Gamma_{i}=\partial \omega=\rho S^{1} .
\end{array}\right.
$$

We deal with the inverse problem of reconstructing the inner circle (i.e. find the radius $\rho$ ) from boundary measurements on the outer circle $\partial \Omega$. We give a result for identifying uniquely the obstacle from two pairs of Cauchy data $\left(f_{1}, \partial_{\mathrm{n}} u_{1}=\left.g_{1}\right|_{\Gamma}\right)$ and $\left(f_{2}, \partial_{\mathrm{n}} u_{2}=\left.g_{2}\right|_{\Gamma}\right)$, where $u_{1}$ and $u_{2}$ are the respective solutions of Problem (3.1) with the Dirichlet data $f_{1}$ and $f_{2}$. Precisely, we show the following result:

Theorem 3.1. Given $\alpha>0$ and $\beta<0$, one can determine uniquely $\Gamma_{i}$ (i.e. the radius $\rho$ ) with two pairs of measurements $\left(f_{1}, g_{1}\right)$ and $\left(f_{2}, g_{2}\right)$, provided $f_{1}$ and $f_{2}$ are suitably chosen.

The idea of the proof is the following. We consider two inputs of the form $f_{1}=$ $R^{n_{1}} \cos \left(n_{1} \theta\right)$ and $f_{2}=R^{n_{2}} \cos \left(n_{2} \theta\right)$. We assume that two inner radii $\rho$ and $\widetilde{\rho}$ generate the same Cauchy data for both inputs. We obtain a system of equations in $t:=\rho / \widetilde{\rho}$ with respect to $n_{1}$ and $n_{2}$ of the kind

$$
h_{n_{i}}^{1}(t)=h_{n_{i}}^{2}(t) \quad \forall i=1,2,
$$

where the $h_{n_{i}}^{i}$ are real valued functions of the real variable. We will show that for $n_{1}$ and $n_{2}$ suitably chosen, the unique solution of the previous system is $t=1$ meaning $\rho=\widetilde{\rho}$.

Proof. In the sequel, the couple $(r, \theta)$ represents the polar coordinates. Moreover, $a_{n}(f)$ and $b_{n}(f)$ (respectively $a_{n}(g)$ and $b_{n}(g)$ ) represent the respective Fourier coefficients of $f$ and $g$. We subdivide the proof in two parts.

First step: preliminary computations. Assume that $(f, g)$ is a Cauchy pair for Problem (3.1) set in the annulus $B_{R} \backslash \overline{B_{\rho}}$. Its solution is of the following form

$$
u(r, \theta)=a_{-1} \ln r+a_{0}+\sum_{n=1}^{\infty}\left(a_{n} r^{n}+a_{-n} r^{-n}\right) \cos (n \theta)+\sum_{n=1}^{\infty}\left(b_{n} r^{n}+b_{-n} r^{-n}\right) \sin (n \theta)
$$

where, for $n \geq 1$,

$$
\left\{\begin{array}{ccc}
a_{n} R^{n}+a_{-n} R^{-n} & =a_{n}(f) \\
a_{n} R^{n}-a_{-n} R^{-n} & =\frac{R}{n} a_{n}(g) \\
a_{n} \rho^{n}\left(\frac{-\beta n^{2}}{\rho^{2}}-\frac{n}{\rho}+\alpha\right)+a_{-n} \rho^{-n}\left(\frac{-\beta n^{2}}{\rho^{2}}+\frac{n}{\rho}+\alpha\right) & =0
\end{array}\right.
$$

(and with the same system replacing $a_{n}$ by $b_{n}$ ). Since the matrix of the linear system is of rank two, we get the relation

$$
2 a_{n}(f) X_{n}(\rho)=\left(\frac{R^{2 n}}{\rho^{2 n}} Y_{n}(\rho)-X_{n}(\rho)\right)\left(\frac{R}{n} a_{n}(g)-a_{n}(f)\right)
$$

where we set

$$
X_{n}(\rho):=\frac{-\beta n^{2}}{\rho^{2}}-\frac{n}{\rho}+\alpha \quad \text { and } \quad Y_{n}(\rho):=\frac{-\beta n^{2}}{\rho^{2}}+\frac{n}{\rho}+\alpha .
$$


Hence,

$$
\frac{2 a_{n}(f)}{\frac{R}{n} a_{n}(g)-a_{n}(f)}=\frac{\frac{R^{2 n}}{\rho^{2 n}} Y_{n}(\rho)-X_{n}(\rho)}{X_{n}(\rho)} .
$$

Notice that the left hand side does not depend on the inner radius $\rho$.

Second step: derivation of an equation using two measurements. Assume now that $\left(f=R^{n} \cos (n \theta), g\right)$ is a Cauchy pair for the boundary value problem (3.1) in the annuli $B_{R} \backslash \overline{B_{\rho}}$ and $B_{R} \backslash \overline{B_{\widetilde{\rho}}}$ (where $0<\widetilde{\rho}<R$ ). The previous remark provides the relation

$$
\frac{\frac{R^{2 n}}{\rho^{2 n}} Y_{n}(\rho)-X_{n}(\rho)}{X_{n}(\rho)}=\frac{\frac{R^{2 n}}{\widetilde{\rho}^{2 n}} Y_{n}(\widetilde{\rho})-X_{n}(\widetilde{\rho})}{X_{n}(\widetilde{\rho})} .
$$

Hence

$$
\frac{\widetilde{\rho}^{2 n}}{\rho^{2 n}} Y_{n}(\rho) X_{n}(\widetilde{\rho})=Y_{n}(\widetilde{\rho}) X_{n}(\rho)
$$

Setting $t:=\frac{\widetilde{\rho}}{\rho}$, we then get

$$
t^{2 n} \frac{-\beta n^{2}-n t \rho+\alpha t^{2} \rho^{2}}{-\beta n^{2}-n \rho+\alpha \rho^{2}}=\frac{-\beta n^{2}+n t \rho+\alpha t^{2} \rho^{2}}{-\beta n^{2}+n \rho+\alpha \rho^{2}} .
$$

Denoting $b_{n}:=\frac{n \rho+\beta n^{2}-\alpha \rho^{2}}{n \rho-\beta n^{2}+\alpha \rho^{2}}$, we check

$$
1+\frac{2 R}{\beta} \leq b_{n}=1-\frac{2 n \rho}{n \rho-\beta n^{2}+\alpha \rho^{2}} \leq 1, \forall n \in \mathbb{N}
$$

and see that $t$ is solution of

$$
t^{2 n} \frac{n t \rho+\beta n^{2}-\alpha t^{2} \rho^{2}}{n t \rho-\beta n^{2}+\alpha t^{2} \rho^{2}}=b_{n}
$$

or equivalently

$$
t^{2 n} \frac{1+\beta \frac{n}{t \rho}-\alpha \frac{t \rho}{n}}{1-\beta \frac{n}{t \rho}+\alpha \frac{t \rho}{n}}=b_{n} .
$$

Setting $P_{n}(t):=-\beta \frac{n}{t \rho}+\alpha \frac{t \rho}{n}$, this gives

$$
t^{2 n}\left(1-P_{n}(t)\right)=b_{n}\left(1+P_{n}(t)\right)
$$

and then the equation in $t$ at fixed $\rho$

$$
\frac{t^{2 n}-b_{n}}{t^{2 n}+b_{n}}=-\beta \frac{n}{t \rho}+\alpha \frac{t \rho}{n}
$$

If there are two distinct inner radii $\rho$ and $\widetilde{\rho}$ such that the measurements $g_{i}$ are the same for the annuli $B_{R} \backslash \overline{B_{\rho}}$ and $B_{R} \backslash \overline{B_{\widetilde{\rho}}}$, then Equation $(3.2$ has at least two solutions one of 
which is 1 . We will prove that when we fix $n_{1}$ and choose $n_{2}$ sufficiently large, the only common solution of

$$
\frac{t^{2 n_{i}}-b_{n_{i}}}{t^{2 n_{i}}+b_{n_{i}}}=-\beta \frac{n_{i}}{t \rho}+\alpha \frac{t \rho}{n_{i}} \quad i=1,2
$$

is $t=1$ and then $\widetilde{\rho}=\rho$. This means that only two measurements are necessary for the identifiability.

Third step: the identifiability proof. We see the solution of Equation 3.2 as the intersection in $\mathbb{R}^{+}$of the two curves $\left(C_{1}\right)$ and $\left(C_{2}\right)$ where

$$
\left(C_{1}\right): t \mapsto h_{n}^{1}(t):=\frac{t^{2 n}-b_{n}}{t^{2 n}+b_{n}} \quad \text { and } \quad\left(C_{2}\right): t \mapsto h_{n}^{2}(t):=-\beta \frac{n}{t \rho}+\alpha \frac{t \rho}{n} \geq-\frac{\beta}{t R} n .
$$

Fix $n_{1}=1$, then Equation $(3.2)$ is polynomial of degree 4, it has at most four solutions in $\mathbb{R}$, one of them is 1 . Set $\mathcal{S}$ the set of positive real solutions of $(3.2)$ distinct of 1 . It has at most three elements.

If $\mathcal{S} \neq \emptyset$, take $t \in \mathcal{S}$ and consider the sequences $h_{n}^{i}(t)$ for $i=1,2$. The sequence $h_{n}^{2}(t)$ clearly goes to infinity. For the sequence $h_{n}^{1}(t)$, we have to distinguish the case $t>1$ and $t<1$. If $t>1$, then $h_{n}^{1}(t)$ tends to 1 since

$$
\left|h_{n}^{1}(t)-1\right|=\left|\frac{2 b_{n}}{t^{2 n}+b_{n}}\right| \leq\left|\frac{2}{t^{2 n}+1+\frac{2 R}{\beta}}\right| .
$$

For $t<1, h_{n}^{1}(t)$ goes to -1 . Hence, from the uniform in $\rho$ bounds in the estimations for these two sequences, there is a rank $n_{t}$, such that for $n \geq n_{t}, t$ is not solution of Equation (3.2). Taking the maximum $N$ of $n_{t}$ for $t \in S$, we conclude that, for a fixed $n_{1}=1$ and $n_{2} \geq N$, the unique common root of $(3.3)$ is $t=1$ and then that only two boundary Cauchy measurements are sufficient to identify the inner circle.

\section{Existence of shape derivatives}

In the previous section, we have proved an identifiability result in annular configurations. We want now to reconstruct the obstacle by minimizing the least squares functional 2.4 and solve the optimization problem (2.6) using shape optimization methods. Hence, we want to use the notion of shape derivatives. Thus, in this section, we prove that the solution $u$ of Problem (2.5) is twice differentiable with respect to the shape $\omega \in \mathcal{O}_{\delta}$.

Let $\boldsymbol{\theta} \in \mathcal{U}$. We recall that $\mathcal{O}_{\delta}$ is defined by (2.1), $\mathcal{U}$ by (2.7) and that we define $\omega_{\theta}:=(\mathbf{I}+\boldsymbol{\theta})(\omega)$. Then, we consider $u_{\theta} \in \mathrm{H}^{1}\left(\Omega \backslash \overline{\omega_{\theta}}\right)$ solution of

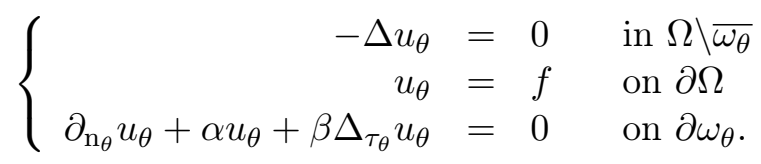

Notice that $u_{\theta}$ belongs to $\mathrm{H}^{3}(\Omega \backslash \bar{\omega})$. Let us consider $F \in \mathrm{H}^{3}(\Omega \backslash \bar{\omega})$ such that

$$
-\Delta F=0 \text { in } \Omega, \quad F=f \text { on } \partial \Omega \text { and } F=0 \text { in } \Omega_{\delta} .
$$

Then, we define

$$
v_{\theta}:=\left(u_{\theta}-F\right) \circ(\mathbf{I}+\boldsymbol{\theta})
$$


and the variational formulation of Problem 4.1 is then given by

$$
\int_{\Omega \backslash \overline{\omega_{\theta}}} \nabla v_{\theta} \cdot \nabla \varphi_{\theta}+\int_{\partial \omega_{\theta}}\left(\alpha v_{\theta} \varphi_{\theta}-\beta \nabla_{\tau_{\theta}} v_{\theta} \cdot \nabla_{\tau_{\theta}} \varphi_{\theta}\right)=\int_{\Omega \backslash \overline{\omega_{\theta}}} \Delta F \varphi_{\theta} \quad \forall \varphi_{\theta} \in \mathrm{H}_{\partial \Omega}^{1}\left(\Omega \backslash \overline{\omega_{\theta}}\right),
$$

since $\int_{\partial \omega_{\theta}}-\beta \Delta_{\tau_{\theta}} v_{\theta} \varphi_{\theta}=\int_{\partial \omega_{\theta}} \beta \nabla_{\tau_{\theta}} v_{\theta} \cdot \nabla_{\tau_{\theta}} \varphi_{\theta}$. We will apply the general method exposed in [21, Chapter 5] using the implicit function theorem on $v_{\theta}$.

\subsection{Characterization of $v_{\theta}$}

Lemma 4.1 (Characterization of $v_{\theta}$ ). For $\boldsymbol{\theta} \in \mathcal{U}$, $v_{\theta}$ satisfies for all $\varphi \in \mathrm{H}_{\partial \Omega}^{1}(\Omega \backslash \bar{\omega})$

$$
\int_{\Omega \backslash \bar{\omega}} \nabla\left(A(\boldsymbol{\theta}) v_{\theta}\right) \cdot \nabla \varphi+\int_{\partial \omega} \alpha v_{\theta} \varphi w_{\theta}-\int_{\partial \omega} \beta\left(C(\boldsymbol{\theta}) \nabla_{\tau} v_{\theta}\right) \cdot \nabla_{\tau} \varphi=\int_{\Omega \backslash \bar{\omega}} \Delta F \varphi J_{\theta}
$$

where

$$
\begin{aligned}
J_{\boldsymbol{\theta}} & :=\operatorname{det}(\mathrm{I}+\nabla \boldsymbol{\theta}) \in \mathrm{W}^{2, \infty}\left(\overline{\Omega_{\delta}}\right), \\
w_{\theta} & :=\operatorname{det}(\mathrm{I}+\nabla \boldsymbol{\theta})\left\|t(\mathrm{I}+\nabla \boldsymbol{\theta})^{-1} \mathbf{n}\right\| \in \mathrm{W}^{2, \infty}\left(\overline{\Omega_{\delta}}\right), \\
A(\boldsymbol{\theta}) & :=\nu J_{\boldsymbol{\theta}}(\mathrm{I}+\nabla \boldsymbol{\theta})^{-1}\left(\mathrm{I}+{ }^{t} \nabla \boldsymbol{\theta}\right)^{-1} \in \mathrm{W}^{2, \infty}\left(\overline{\Omega_{\delta}}, \mathcal{M}_{d, d}\right), \\
C(\boldsymbol{\theta}) & :=w_{\theta}(\mathrm{I}+\nabla \boldsymbol{\theta})^{-1}{ }^{t}(\mathrm{I}+\nabla \boldsymbol{\theta})^{-1} \in \mathrm{W}^{2, \infty}\left(\overline{\Omega_{\delta}}, \mathcal{M}_{d, d}\right) .
\end{aligned}
$$

Moreover, $v_{\theta}$ belongs to $\mathrm{H}_{\partial \Omega}^{3}(\Omega \backslash \bar{\omega})$.

Remark 4.2. Hence, we characterize $v_{\theta}$ as the solution of

$$
\left\{\begin{aligned}
-\operatorname{div}\left(A(\boldsymbol{\theta}) \nabla v_{\theta}\right) & =\Delta F J_{\theta} & & \text { in } \Omega \backslash \bar{\omega} \\
v_{\theta} & =0 & & \text { on } \partial \Omega \\
\left(A(\boldsymbol{\theta}) \nabla v_{\theta}\right) \cdot \mathbf{n}+\alpha v_{\theta} w_{\theta}+\beta \operatorname{div}_{\tau}\left(C(\boldsymbol{\theta}) \nabla_{\tau} v_{\theta}\right) & =0 & & \text { on } \partial \omega .
\end{aligned}\right.
$$

Proof. Let $\varphi \in \mathrm{H}_{\partial \Omega}^{1}(\Omega \backslash \bar{\omega})$. We define

$$
\varphi_{\theta}:=\varphi \circ(\mathbf{I}+\boldsymbol{\theta})^{-1} \in \mathrm{H}_{\partial \Omega}^{1}\left(\Omega \backslash \overline{\omega_{\theta}}\right) \quad \text { and } \quad T(\boldsymbol{\theta}):=\left(\mathrm{I}+{ }^{t} \nabla \boldsymbol{\theta}\right)^{-1} \in \mathrm{W}^{2, \infty}\left(\mathbb{R}^{d}, \mathcal{M}_{d, d}\right) .
$$

Thus, using $\varphi_{\theta}$ as a test function in the variational formulation 4.2 , we obtain

$$
\begin{gathered}
\int_{\Omega \backslash \overline{\omega_{\theta}}}\left[\left(T(\boldsymbol{\theta}) \nabla v_{\theta}\right) \circ(\mathbf{I}+\boldsymbol{\theta})^{-1}\right] \cdot\left[(T(\boldsymbol{\theta}) \nabla \varphi) \circ(\mathbf{I}+\boldsymbol{\theta})^{-1}\right] \\
+\int_{\partial \omega_{\theta}} \alpha\left[v_{\theta} \circ(\mathbf{I}+\boldsymbol{\theta})^{-1}\right]\left[\varphi \circ(\mathbf{I}+\boldsymbol{\theta})^{-1}\right] \\
-\int_{\partial \omega_{\theta}}\left\{\beta\left[\left(T(\boldsymbol{\theta}) \nabla v_{\theta}\right) \circ(\mathbf{I}+\boldsymbol{\theta})^{-1}-\left(\mathbf{n} \circ(\mathbf{I}+\boldsymbol{\theta})^{-1}\right) \cdot\left(\left(\left(T(\boldsymbol{\theta}) \nabla v_{\theta}\right) \circ(\mathbf{I}+\boldsymbol{\theta})^{-1}\right) \cdot\left(\mathbf{n} \circ(\mathbf{I}+\boldsymbol{\theta})^{-1}\right)\right)\right]\right. \\
\left.\cdot\left[(T(\boldsymbol{\theta}) \nabla \varphi) \circ(\mathbf{I}+\boldsymbol{\theta})^{-1}-\left(\mathbf{n} \circ(\mathbf{I}+\boldsymbol{\theta})^{-1}\right) \cdot\left(\left((T(\boldsymbol{\theta}) \nabla \varphi) \circ(\mathbf{I}+\boldsymbol{\theta})^{-1}\right) \cdot\left(\mathbf{n} \circ(\mathbf{I}+\boldsymbol{\theta})^{-1}\right)\right)\right]\right\} \\
\quad=\int_{\Omega \backslash \overline{\omega_{\theta}}} \Delta F\left(\varphi \circ(\mathbf{I}+\boldsymbol{\theta})^{-1}\right) .
\end{gathered}
$$

Hence, using the change of variables $x=(\mathbf{I}+\boldsymbol{\theta})(y)$, we obtain

$$
\begin{aligned}
\int_{\Omega \backslash \bar{\omega}} & \left(A(\boldsymbol{\theta}) \nabla v_{\theta}\right) \cdot \nabla \varphi+\int_{\partial \omega} \alpha v_{\theta} \varphi w_{\theta} \\
& -\int_{\partial \omega} \beta\left[T(\boldsymbol{\theta}) \nabla v_{\theta}-\mathbf{n}\left(T(\boldsymbol{\theta}) \nabla v_{\theta} \cdot \mathbf{n}\right)\right] \cdot[T(\boldsymbol{\theta}) \nabla \varphi-\mathbf{n}(T(\boldsymbol{\theta}) \nabla \varphi \cdot \mathbf{n})] w_{\theta}=\int_{\Omega \backslash \bar{\omega}} \Delta F \varphi J_{\theta} .
\end{aligned}
$$


Therefore, we obtain the announced result. Notice that $J_{\theta}$ is the volume Jacobian and $w_{\theta}$ is the surface Jacobian.

\subsection{Differentiability of $\boldsymbol{\theta} \mapsto v_{\theta}$}

Lemma 4.3 (Differentiability of $\boldsymbol{\theta} \mapsto v_{\theta}$ ). The function

$$
\boldsymbol{\theta} \in \mathcal{U} \mapsto v_{\theta} \in \mathrm{H}_{\partial \Omega}^{2}(\Omega \backslash \bar{\omega})
$$

is differentiable in a neighborhood of $\mathbf{0}$ (and even $C^{\infty}$ ).

Proof. Let us consider the application the application $\mathcal{F}: \mathcal{U} \times \mathrm{H}_{\partial \Omega}^{2}(\Omega \backslash \bar{\omega}) \rightarrow \mathrm{L}^{2}(\Omega \backslash \bar{\omega})$ defined for all $\varphi \in \mathrm{H}_{\partial \Omega}^{1}(\Omega \backslash \bar{\omega})$ by

$$
\langle\mathcal{F}(\boldsymbol{\theta}, v), \varphi\rangle=\int_{\Omega \backslash \bar{\omega}}(A(\boldsymbol{\theta}) \nabla v) \cdot \nabla \varphi+\int_{\partial \omega} \alpha v \varphi w_{\theta}-\int_{\partial \omega} \beta\left(C(\theta) \nabla_{\tau} v\right) \cdot \nabla_{\tau} \varphi-\int_{\Omega \backslash \bar{\omega}} \Delta F \varphi J_{\theta} .
$$

We have, for $\boldsymbol{\theta}=\mathbf{0}, \mathcal{F}\left(\mathbf{0}, v_{0}\right)=\mathcal{F}(\mathbf{0}, u-F)=0$. Moreover, we prove analogously to what is done in [21, Proof of Theorem 5.3.2] that the application $\mathcal{F}$ is $C^{\infty}$. Finally, we compute for all $w, \hat{w} \in \mathrm{H}_{\partial \Omega}^{1}(\Omega \backslash \bar{\omega})$,

$$
\mathrm{D}_{u} \mathcal{F}(\mathbf{0}, u-F)(w) \cdot \hat{w}=\int_{\Omega \backslash \bar{\omega}} \nabla w \cdot \nabla \hat{w}+\int_{\partial \omega} \alpha w \hat{w}-\int_{\partial \omega} \beta \nabla_{\tau} w \cdot \nabla_{\tau} \hat{w} .
$$

Thus, $\mathrm{D}_{u} \mathcal{F}(\mathbf{0}, u-F)$ is an isomorphism.

Hence, the implicit function theorem applies and then there exists a $C^{\infty}$ function $\boldsymbol{\theta} \in \mathcal{U} \mapsto v(\boldsymbol{\theta}) \in \mathrm{H}_{\partial \Omega}^{2}(\Omega \backslash \bar{\omega})$ such that $\mathcal{F}(\mathbf{0}, v(\boldsymbol{\theta}))=0$ in a neighborhood of $\mathbf{0}$. Using the uniqueness of the solution of such a problem, we obtain that $\boldsymbol{\theta} \in \mathcal{U} \mapsto v_{\theta} \in \mathrm{H}_{\partial \Omega}^{2}(\Omega \backslash \bar{\omega})$ is $C^{\infty}$.

\subsection{Existence of shape derivatives}

Lemma 4.4 (Differentiability of $\boldsymbol{\theta} \mapsto u_{\theta}$ ). There exists $\widetilde{u}_{\theta}$ an extension in $\Omega$ of $u_{\theta} \in$ $\mathrm{H}^{1}\left(\Omega \backslash \overline{\omega_{\theta}}\right)$ such that

$$
\boldsymbol{\theta} \in \mathcal{U} \mapsto \widetilde{u}_{\theta} \in \mathrm{H}^{1}(\Omega)
$$

is differentiable at $\mathbf{0}$ (and even $C^{\infty}$ in a neighborhood of $\mathbf{0}$ ).

Proof. For $\boldsymbol{\theta} \in \mathcal{U}, u_{\theta}=v_{\theta} \circ(\mathbf{I}+\boldsymbol{\theta})^{-1} \in \mathrm{H}_{\partial \Omega}^{2}\left(\Omega \backslash \overline{\omega_{\theta}}\right)$. According to the differentiability of $\boldsymbol{\theta} \mapsto v_{\theta}$ (see Lemma 4.3) and Stein's extension theorem (see for example [1, Theorem 5.24]), there exists $\widetilde{v}_{\theta}$, an extension of $v_{\theta}$, such that $\boldsymbol{\theta} \in \mathcal{U} \mapsto \widetilde{v}_{\theta} \in \mathrm{H}_{\partial \Omega}^{2}(\Omega)$ is differentiable at $\mathbf{0}$. Moreover,

$$
\boldsymbol{\theta} \in \mathcal{U} \mapsto(\mathbf{I}+\boldsymbol{\theta})^{-1}-\mathbf{I} \in \mathbf{W}^{2, \infty}\left(\mathbb{R}^{d}\right)
$$

is differentiable at $\mathbf{0}$. Thus,

$$
\varphi_{1}: \boldsymbol{\theta} \in \mathcal{U} \mapsto\left(v_{\theta},(\mathbf{I}+\boldsymbol{\theta})^{-1}-\mathbf{I}\right) \in \mathrm{H}_{\partial \Omega}^{2}(\Omega) \times \mathbf{W}^{2, \infty}\left(\mathbb{R}^{d}\right)
$$

is differentiable at $\mathbf{0}$. We apply [21, Lemma 5.3.9] to get that

$$
\varphi_{2}:(g, \boldsymbol{\mu}) \in \mathrm{H}^{2}\left(\mathbb{R}^{d}\right) \times \mathbf{W}^{2, \infty}(\Omega) \mapsto g \circ(\mathbf{I}+\boldsymbol{\mu}) \in \mathrm{H}^{1}\left(\mathbb{R}^{d}\right)
$$

is $C^{1}$ in a neighborhood of $\mathbf{0}$. By composition, $\boldsymbol{\theta} \in \mathcal{U} \mapsto \widetilde{u}_{\theta}:=\varphi_{2} \circ \varphi_{1}(\boldsymbol{\theta}) \in \mathrm{H}_{\partial \Omega}^{1}(\Omega)$ is differentiable at $\mathbf{0}$. 
Using the Fréchet differentiability given by this lemma, we obtain the Gâteaux differentiability in the direction $\boldsymbol{V} \in \mathcal{U}$ : there exist $u_{t}$, extension in $\Omega$ of $u_{t}$ such that the function $t \in[0, T) \mapsto u_{t} \in \mathrm{H}^{1}(\Omega)$ is differentiable at 0 by composition. Hence, we prove the following result:

Lemma 4.5. The solution $u$ of Problem 2.5 is differentiable with respect to the domain $\omega \in \mathcal{O}_{\delta}$. We denote by $u^{\prime}$ its derivative at 0 .

Proceeding in the same way (using $\mathrm{H}_{\partial \Omega}^{3}(\Omega \backslash \bar{\omega})$ instead of $\mathrm{H}_{\partial \Omega}^{2}(\Omega \backslash \bar{\omega})$ and $\mathbf{W}^{3, \infty}\left(\mathbb{R}^{d}\right)$ instead of $\mathbf{W}^{2, \infty}\left(\mathbb{R}^{d}\right)$ ), we prove the following result:

Proposition 4.6. The solution $u$ of Problem (2.5) is twice differentiable with respect to the domain $\omega \in \mathcal{O}_{\delta}$. We denote by $u^{\prime \prime}$ its derivative at 0 .

\section{Shape calculus}

Since we proved in the previous section the differentiability with respect to the domain, we can now compute the shape derivatives of the state and of the coast functional $J$ that we want to minimize. Thus, we aim to make a sensitivity (with respect to the shape) analysis. Then, let us consider the problem on $\Omega \backslash \overline{\omega_{t}}$

$$
\left\{\begin{aligned}
-\Delta u_{t} & =0 & & \text { in } \Omega \backslash \overline{\omega_{t}} \\
u_{t} & =f & & \text { on } \partial \Omega \\
\partial_{\mathrm{n}_{t}} u_{t}+\alpha u_{t}+\beta \Delta_{\tau_{t}} u_{t} & =0 & & \text { on } \partial \omega_{t} .
\end{aligned}\right.
$$

Let us remain the definition of the shape derivative in our situation (see [21] for details).

- If the mapping $\boldsymbol{\theta} \in \mathcal{U} \mapsto u_{\theta} \circ(\mathbf{I}+\boldsymbol{\theta}) \in \mathrm{H}^{1}(\Omega \backslash \bar{\omega})$ is Fréchet differentiable at $\mathbf{0}$, we say that $\boldsymbol{\theta} \mapsto u_{\theta}$ possesses a total first variation (or derivative) at $\mathbf{0}$. In such a case, this total first derivative at $\mathbf{0}$ in the direction $\boldsymbol{\theta}$ is denoted by $\dot{u}_{\theta}$.

- If, for every $\mathscr{D} \subset \subset \Omega \backslash \bar{\omega}$, the mapping $\boldsymbol{\theta} \in \mathcal{U} \mapsto u_{\theta \mid \mathscr{D}} \in \mathrm{H}^{1}(\mathscr{D})$ is Fréchet differentiable at $\mathbf{0}$, we say that $\boldsymbol{\theta} \mapsto u_{\theta}$ possesses a local first variation (or derivative) at $\mathbf{0}$. In such a case, this local first derivative at $\mathbf{0}$ in the direction $\boldsymbol{\theta}$ is denoted by $u_{\theta}^{\prime}$, is called shape derivative and is well defined in the whole domain $\Omega \backslash \bar{\omega}$ :

$$
u_{\theta}^{\prime}=\left.\frac{d}{d t}\left(u_{t \boldsymbol{\theta} \mid \mathscr{D}}\right)\right|_{t=0} \quad \text { in each } \mathscr{D} \subset \subset \Omega \backslash \bar{\omega} .
$$

We define similarly the higher order shape derivative. In the following, for $\boldsymbol{V} \in \mathcal{U}$, we denote by $u^{\prime}$ the local first variation $u_{V}^{\prime}$ which is referred as the shape derivative of the state.

We first characterize the shape derivative of the state differentiating with respect to the domain the Ventcel boundary conditions. Then, using this characterization, we compute the shape gradient of the functional $J$ and obtain a formula with an explicit dependance with respect to the perturbation direction $\boldsymbol{V}$ introducing an adapted adjoint problem. We also characterize the shape Hessian and finally prove the instability of our shape optimization problem (2.6) using a local regularity argument. 


\subsection{Shape derivative of the problem}

Proposition 5.1 (First order shape derivative of the state). Let $\boldsymbol{V} \in \mathcal{U}$. The shape derivative $u^{\prime}$ of $u$ which belongs to $\mathrm{H}^{1}(\Omega \backslash \bar{\omega})$ is the only solution of the following boundary values problem

$$
\left\{\begin{aligned}
-\Delta u^{\prime} & =0 & & \text { in } \Omega \backslash \bar{\omega} \\
u^{\prime} & =0 & & \text { on } \partial \Omega \\
\partial_{\mathrm{n}} u^{\prime}+\alpha u^{\prime}+\beta \Delta_{\tau} u^{\prime} & =\xi\left(u, V_{\mathrm{n}}\right) & & \text { on } \partial \omega
\end{aligned}\right.
$$

with

$$
\begin{aligned}
\xi\left(u, V_{\mathrm{n}}\right):=V_{\mathrm{n}}\left(-\alpha \partial_{\mathrm{n}} u-\alpha \mathrm{H} u\right. & \left.+\Delta_{\tau} u\right)+\nabla_{\tau} u \cdot \nabla_{\tau} V_{\mathrm{n}} \\
& -\beta \Delta_{\tau}\left(V_{\mathrm{n}} \partial_{\mathrm{n}} u\right)-\beta \operatorname{div}_{\tau}\left(V_{\mathrm{n}} \mathrm{H} \nabla_{\tau} u-2 V_{\mathrm{n}} \mathrm{D}^{2} b \nabla_{\tau} u\right),
\end{aligned}
$$

where $\mathrm{H}$ is the mean curvature of $\partial \omega$ and $b$ is the signed distance.

We refer to Appendix B where we state some useful results concerning shape differential calculus (in particular concerning the signed distance $b$ ).

Proof. For $t \in[0, T)$, we consider Problem (5.1). By differentiating with respect to the shape at $t=0$, we classically obtain (see for example [21, Chapter 5])

$$
-\Delta u^{\prime}=0 \quad \text { in } \Omega \backslash \bar{\omega} \quad \text { and } \quad u^{\prime}=0 \quad \text { on } \partial \Omega .
$$

Now let us compute the shape derivative of the Ventcel boundary condition $\partial_{\mathrm{n}_{t}} u_{t}+$ $\alpha u_{t}+\beta \Delta_{\tau_{t}} u_{t}=0$ on $\partial \omega_{t}$ at $t=0$. Let $\varphi \in \mathrm{H}^{2}(\Omega \backslash \bar{\omega})$ compactly supported in $\Omega_{\delta}$. Using Stein's extension theorem, $\varphi$ admits an extension in $\mathrm{H}^{2}\left(\mathbb{R}^{d}\right)$ still denoted by $\varphi$ and which is still compactly supported in $\Omega_{\delta}$. This extension belongs in particular to $\mathrm{H}^{2}\left(\Omega \backslash \overline{\omega_{t}}\right)$. Using $\varphi$ as a test function in the variational formulation of Problem (5.1), we have

$$
\int_{\Omega \backslash \overline{\omega_{t}}} \nabla u_{t} \cdot \nabla \varphi+\int_{\partial \omega_{t}}\left(\alpha u_{t} \varphi-\beta \nabla_{\tau_{t}} u_{t} \cdot \nabla_{\tau_{t}} \varphi\right)=\int_{\Omega \backslash \overline{\omega_{t}}} \Delta F \varphi .
$$

Then, by using Hadamard formulas (on the volume and on the surface: see [21, Proposition 5.4.4 and Proposition 5.4.18]), we obtain

$$
\begin{aligned}
\int_{\Omega \backslash \bar{\omega}} \nabla u^{\prime} \cdot & \nabla \varphi+\int_{\partial \omega} \nabla u \cdot \nabla \varphi V_{\mathrm{n}} \\
& +\int_{\partial \omega}\left[\alpha u^{\prime} \varphi-\left.\beta \frac{d}{d t}\left(\nabla_{\tau_{t}} u_{t}\right)\right|_{t=0} \cdot \nabla_{\tau} \varphi-\left.\beta \nabla_{\tau} u \cdot \frac{d}{d t}\left(\nabla_{\tau_{t}} \varphi\right)\right|_{t=0}\right. \\
+ & \left.V_{\mathrm{n}}\left(\partial_{\mathrm{n}}\left(\alpha u \varphi-\beta \nabla_{\tau} u \cdot \nabla_{\tau} \varphi\right)+\mathrm{H}\left(\alpha u \varphi-\beta \nabla_{\tau} u \cdot \nabla_{\tau} \varphi\right)\right)\right]=\int_{\partial \omega}(\Delta F \varphi) V_{\mathrm{n}}
\end{aligned}
$$

Moreover,

$$
\begin{aligned}
\left.\frac{d}{d t}\left(\nabla_{\tau_{t}} u_{t}\right)\right|_{t=0} & =\left.\frac{d}{d t}\left(\nabla u_{t}-\left(\nabla u_{t} \cdot \mathbf{n}_{t}\right) \mathbf{n}_{t}\right)\right|_{t=0} \\
& =\nabla_{\tau} u^{\prime}+\left(\nabla u \cdot \nabla_{\tau} V_{\mathrm{n}}\right) \mathbf{n}+\partial_{\mathrm{n}} u \nabla_{\tau} V_{\mathrm{n}}
\end{aligned}
$$


using the fact that $\mathbf{n}^{\prime}=-\nabla_{\tau} V_{\mathrm{n}}$ (see Proposition B.1). Hence, using the same result for $\left.\frac{d}{d t}\left(\nabla_{\tau_{t}} \varphi\right)\right|_{t=0}$, we obtain

$$
\begin{aligned}
\int_{\Omega \backslash \bar{\omega}} \nabla u^{\prime} \cdot \nabla \varphi & +\int_{\partial \omega} \nabla u \cdot \nabla \varphi V_{\mathrm{n}} \\
+ & \int_{\partial \omega}\left[\alpha u^{\prime} \varphi-\beta\left(\nabla_{\tau} u^{\prime}+\partial_{\mathrm{n}} u \nabla_{\tau} V_{\mathrm{n}}+\left(\nabla u \cdot \nabla_{\tau} V_{\mathrm{n}}\right) \mathbf{n}\right) \cdot \nabla_{\tau} \varphi\right. \\
& \quad-\beta \nabla_{\tau} u \cdot\left(\partial_{\mathrm{n}} \varphi \nabla_{\tau} V_{\mathrm{n}}+\left(\nabla \varphi \cdot \nabla_{\tau} V_{\mathrm{n}}\right) \mathbf{n}\right) \\
+ & \left.V_{\mathrm{n}}\left(\partial_{\mathrm{n}}\left(\alpha u \varphi-\beta \nabla_{\tau} u \cdot \nabla_{\tau} \varphi\right)+\mathrm{H}\left(\alpha u \varphi-\beta \nabla_{\tau} u \cdot \nabla_{\tau} \varphi\right)\right)\right]=\int_{\partial \omega}(\Delta F \varphi) V_{\mathrm{n}} .
\end{aligned}
$$

Since $\nabla_{\tau} \varphi \cdot \mathbf{n}=0$ and $\nabla_{\tau} u \cdot \mathbf{n}=0$, this equality writes

$$
\begin{aligned}
\int_{\Omega \backslash \omega} \nabla u^{\prime} \cdot \nabla \varphi & +\int_{\partial \omega} \nabla u \cdot \nabla \varphi V_{\mathrm{n}} \\
& \quad+\int_{\partial \omega}\left[\alpha u^{\prime} \varphi-\beta\left(\nabla_{\tau} u^{\prime}+\partial_{\mathrm{n}} u \nabla_{\tau} V_{\mathrm{n}}\right) \cdot \nabla_{\tau} \varphi-\beta \nabla_{\tau} u \cdot\left(\partial_{\mathrm{n}} \varphi \nabla_{\tau} V_{\mathrm{n}}\right)\right. \\
& \left.+V_{\mathrm{n}}\left(\partial_{\mathrm{n}}\left(\alpha u \varphi-\beta \nabla_{\tau} u \cdot \nabla_{\tau} \varphi\right)+\mathrm{H}\left(\alpha u \varphi-\beta \nabla_{\tau} u \cdot \nabla_{\tau} \varphi\right)\right)\right]=\int_{\partial \omega}(\Delta F \varphi) V_{\mathrm{n}} .
\end{aligned}
$$

We also have using Problem (5.1)

$$
\int_{\Omega \backslash \overline{\omega_{t}}} \nabla u_{t} \cdot \nabla \varphi-\int_{\partial \omega_{t}} \partial_{\mathrm{n}_{t}} u_{t} \varphi=\int_{\Omega \backslash \overline{\omega_{t}}} \Delta F \varphi .
$$

Thus, using Hadamard formulas, we obtain

$$
\begin{aligned}
\int_{\Omega \backslash \bar{\omega}} & \nabla u^{\prime} \cdot \nabla \varphi+\int_{\partial \omega} \nabla u \cdot \nabla \varphi V_{\mathrm{n}} \\
& -\int_{\partial \omega}\left[\partial_{\mathrm{n}} u^{\prime} \varphi-\left(\nabla u \cdot \nabla_{\tau} V_{\mathrm{n}}\right) \varphi+V_{\mathrm{n}}\left(\partial_{\mathrm{n}}\left(\partial_{\mathrm{n}} u \varphi\right)+\partial_{\mathrm{n}} u \varphi \mathrm{H}\right)\right]=\int_{\partial \omega}(\Delta F \varphi) V_{\mathrm{n}} .
\end{aligned}
$$

Subtracting (5.4) and (5.5), we obtain

$$
\begin{aligned}
& \int_{\partial \omega}\left(\partial_{\mathrm{n}} u^{\prime}+\alpha u^{\prime}+\beta \Delta_{\tau} u^{\prime}\right) \varphi= \int_{\partial \omega} \\
&\left.-V_{\mathrm{n}}\left(\partial_{\mathrm{n}}\left(\alpha u \varphi-\beta \nabla_{\tau} u \cdot \nabla_{\tau} \varphi\right)+\mathrm{H}\left(\alpha u \varphi-\beta \nabla_{\tau} u \cdot \nabla_{\tau} \varphi\right)\right)\right] \\
& \quad-\int_{\partial \omega}\left[-\left(\nabla u \cdot \nabla_{\tau} V_{\mathrm{n}}\right) \varphi+V_{\mathrm{n}}\left(\partial_{\mathrm{n}}\left(\partial_{\mathrm{n}} u \varphi\right)+\partial_{\tau} V_{\mathrm{n}}\right) \varphi+\beta \nabla_{\tau} u \cdot\left(\partial_{\mathrm{n}} \varphi \nabla_{\tau} V_{\mathrm{n}}\right)\right] .
\end{aligned}
$$

Since $\partial_{\mathrm{n}}\left(\partial_{\mathrm{n}} u \varphi\right)=\partial_{\mathrm{n}} u \partial_{\mathrm{n}} \varphi+\partial_{\mathrm{nn}}^{2} u \varphi$, this leads

$$
\begin{gathered}
\int_{\partial \omega}\left(\partial_{\mathrm{n}} u^{\prime}+\alpha u^{\prime}+\beta \Delta_{\tau} u^{\prime}\right) \varphi=\int_{\partial \omega}\left[-\beta \operatorname{div}_{\tau}\left(\partial_{\mathrm{n}} u \nabla_{\tau} V_{\mathrm{n}}\right) \varphi+\beta \partial_{\mathrm{n}} \varphi \nabla_{\tau} u \cdot \nabla_{\tau} V_{\mathrm{n}}\right. \\
\left.-V_{\mathrm{n}}\left(\alpha \partial_{\mathrm{n}} u \varphi+\alpha u \partial_{\mathrm{n}} \varphi-\beta \partial_{\mathrm{n}}\left(\nabla_{\tau} u \cdot \nabla_{\tau} \varphi\right)+\mathrm{H} \alpha u \varphi-\mathrm{H} \beta \nabla_{\tau} u \cdot \nabla_{\tau} \varphi\right)\right] \\
-\int_{\partial \omega}\left[-\left(\nabla u \cdot \nabla_{\tau} V_{\mathrm{n}}\right) \varphi+V_{\mathrm{n}}\left(\partial_{\mathrm{n}} u \partial_{\mathrm{n}} \varphi+\partial_{\mathrm{nn}}^{2} u \varphi+\mathrm{H} \partial_{\mathrm{n}} u \varphi\right)\right] .
\end{gathered}
$$


Using an integration by part and the decomposition of the Laplace-Beltrami operator (see for example [21, Proposition 5.4.12]) given by

$$
\Delta u=\Delta_{\tau} u+\mathrm{H} \partial_{\mathrm{n}} u+\partial_{\mathrm{nn}}^{2} u \quad \text { on } \partial \omega,
$$

we obtain

$$
\begin{gathered}
\int_{\partial \omega}\left(\partial_{\mathrm{n}} u^{\prime}+\alpha u^{\prime}+\beta \Delta_{\tau} u^{\prime}\right) \varphi=\int_{\partial \omega}\left[-\beta \operatorname{div}_{\tau}\left(\partial_{\mathrm{n}} u \nabla_{\tau} V_{\mathrm{n}}\right) \varphi-\beta \operatorname{div}_{\tau}\left(\partial_{\mathrm{n}} \varphi \nabla_{\tau} u\right) V_{\mathrm{n}}\right. \\
-V_{\mathrm{n}}\left(\alpha \partial_{\mathrm{n}} u+\alpha \mathrm{H} u-\Delta_{\tau} u\right) \varphi-V_{\mathrm{n}}\left(\alpha u \partial_{\mathrm{n}} \varphi-\beta \partial_{\mathrm{n}}\left(\nabla_{\tau} u \cdot \nabla_{\tau} \varphi\right)+\partial_{\mathrm{n}} u \partial_{\mathrm{n}} \varphi\right) \\
\left.-\operatorname{div}_{\tau}\left(V_{\mathrm{n}} \mathrm{H} \beta \nabla_{\tau} u\right) \varphi+\left(\nabla u \cdot \nabla_{\tau} V_{\mathrm{n}}\right) \varphi\right] .
\end{gathered}
$$

Since (see Theorem B.2)

$$
\partial_{\mathrm{n}}\left(\nabla_{\tau} u \cdot \nabla_{\tau} \varphi\right)+\mathrm{D}^{2} b \nabla_{\tau} u \cdot \nabla_{\tau} \varphi=\nabla_{\tau}\left(\partial_{\mathrm{n}} u\right) \cdot \nabla_{\tau} \varphi+\nabla_{\tau}\left(\partial_{\mathrm{n}} \varphi\right) \cdot \nabla_{\tau} u
$$

and since $\partial_{\mathrm{n}} u+\alpha u+\beta \Delta_{\tau} u=0$ on $\partial \omega$, we have

$$
\begin{aligned}
& \int_{\partial \omega}\left[-\beta \operatorname{div}_{\tau}\left(\partial_{\mathrm{n}} \varphi \nabla_{\tau} u\right) V_{\mathrm{n}}-V_{\mathrm{n}}\left(\alpha u \partial_{\mathrm{n}} \varphi-\beta \partial_{\mathrm{n}}\left(\nabla_{\tau} u \cdot \nabla_{\tau} \varphi\right)+\partial_{\mathrm{n}} u \partial_{\mathrm{n}} \varphi\right)\right] \\
= & -\int_{\partial \omega}\left[\beta \Delta_{\tau} u \partial_{\mathrm{n}} \varphi+\beta \nabla_{\tau} u \cdot \nabla_{\tau}\left(\partial_{\mathrm{n}} \varphi\right)+\alpha u \partial_{\mathrm{n}} \varphi+\partial_{\mathrm{n}} u \partial_{\mathrm{n}} \varphi-\beta \partial_{\mathrm{n}}\left(\nabla_{\tau} u \cdot \nabla_{\tau} \varphi\right)\right] V_{\mathrm{n}} \\
= & -\int_{\partial \omega}\left[\beta \nabla_{\tau} u \cdot \nabla_{\tau}\left(\partial_{\mathrm{n}} \varphi\right)-\beta \partial_{\mathrm{n}}\left(\nabla_{\tau} u \cdot \nabla_{\tau} \varphi\right)\right] V_{\mathrm{n}} \\
= & -\int_{\partial \omega} \beta\left[-\nabla_{\tau}\left(\partial_{\mathrm{n}} u\right) \cdot \nabla_{\tau} \varphi+\partial_{\mathrm{n}}\left(\nabla_{\tau} u \cdot \nabla_{\tau} \varphi\right)+2 \mathrm{D}^{2} b \nabla_{\tau} u \cdot \nabla_{\tau} \varphi-\partial_{\mathrm{n}}\left(\nabla_{\tau} u \cdot \nabla_{\tau} \varphi\right)\right] V_{\mathrm{n}} \\
= & \int_{\partial \omega} \operatorname{div}_{\tau}\left(\beta V_{\mathrm{n}}\left(-\nabla_{\tau}\left(\partial_{\mathrm{n}} u\right)+2 \mathrm{D}^{2} b \nabla_{\tau} u\right)\right) \varphi .
\end{aligned}
$$

Hence, we finally obtain

$$
\begin{gathered}
\int_{\partial \omega}\left(\partial_{\mathrm{n}} u^{\prime}+\alpha u^{\prime}+\beta \Delta_{\tau} u^{\prime}\right) \varphi=\int_{\partial \omega}\left[-\beta \operatorname{div}_{\tau}\left(\partial_{\mathrm{n}} u \nabla_{\tau} V_{\mathrm{n}}\right) \varphi-V_{\mathrm{n}}\left(\alpha \partial_{\mathrm{n}} u+\alpha \mathrm{H} u-\Delta_{\tau} u\right) \varphi\right. \\
\left.-\operatorname{div}_{\tau}\left(V_{\mathrm{n}} \mathrm{H} \beta \nabla_{\tau} u\right) \varphi+\left(\nabla u \cdot \nabla_{\tau} V_{\mathrm{n}}\right) \varphi+\operatorname{div}_{\tau}\left(\beta V_{\mathrm{n}}\left(-\nabla_{\tau}\left(\partial_{\mathrm{n}} u\right)+2 \mathrm{D}^{2} b \nabla_{\tau} u\right)\right) \varphi\right]
\end{gathered}
$$

Then, using the density in $\mathrm{L}^{2}(\partial \omega)$ of the traces on $\partial \omega$ of functions $\mathrm{H}^{2}(\Omega \backslash \bar{\omega})$ with compact support in $\Omega_{\delta}$, we obtain

$$
\begin{aligned}
\partial_{\mathrm{n}} u^{\prime}+\alpha u^{\prime}+\beta \Delta_{\tau} u^{\prime}=V_{\mathrm{n}} & \left(-\alpha \partial_{\mathrm{n}} u-\alpha \mathrm{H} u+\Delta_{\tau} u\right)+\nabla u \cdot \nabla_{\tau} V_{\mathrm{n}} \\
& -\beta \operatorname{div}_{\tau}\left[\partial_{\mathrm{n}} u \nabla_{\tau} V_{\mathrm{n}}+V_{\mathrm{n}} \mathrm{H} \nabla_{\tau} u+V_{\mathrm{n}} \nabla_{\tau}\left(\partial_{\mathrm{n}} u\right)-2 V_{\mathrm{n}} \mathrm{D}^{2} b \nabla_{\tau} u\right] .
\end{aligned}
$$

Noticing that this equalities can be rewrite as

$$
\begin{aligned}
\partial_{\mathrm{n}} u^{\prime}+\alpha u^{\prime}+\beta \Delta_{\tau} u^{\prime}=V_{\mathrm{n}}\left(-\alpha \partial_{\mathrm{n}} u-\right. & \left.-\alpha \mathrm{H} u+\Delta_{\tau} u\right)+\nabla u \cdot \nabla_{\tau} V_{\mathrm{n}} \\
& -\beta \Delta_{\tau}\left(V_{\mathrm{n}} \partial_{\mathrm{n}} u\right)-\beta \operatorname{div}_{\tau}\left[V_{\mathrm{n}} \mathrm{H} \nabla_{\tau} u-2 V_{\mathrm{n}} \mathrm{D}^{2} b \nabla_{\tau} u\right],
\end{aligned}
$$

we obtain the announced result using the fact that $\nabla u \cdot \nabla_{\tau} V_{\mathrm{n}}=\nabla_{\tau} u \cdot \nabla_{\tau} V_{\mathrm{n}}$ on $\partial \omega$. 


\subsection{Shape derivatives of the functional}

\subsubsection{First order shape derivatives of the functional}

Using the previous characterization of the state, we now compute the shape gradient of the functional $J$ in the following statement:

Proposition 5.2 (First order shape derivative of the functional). For $\boldsymbol{V}$ in $\boldsymbol{U}$, the least squares functional $J$ is differentiable at $\omega$ in the direction $\boldsymbol{V}$ with

$$
\nabla J(\omega) \cdot \boldsymbol{V}=-\int_{\partial \omega}\left[-\alpha \mathrm{H} u w+\left(-\mathrm{I}+\beta\left(\mathrm{HI}-2 \mathrm{D}^{2} b\right)\right)\left(\nabla_{\tau} u \cdot \nabla_{\tau} w\right)+\partial_{\mathrm{n}} u \partial_{\mathrm{n}} w\right] V_{\mathrm{n}}
$$

where $\mathrm{H}$ is the mean curvature of $\partial \omega, b$ is the signed distance and where $w \in \mathrm{H}^{1}(\Omega \backslash \bar{\omega})$ is the solution of the following boundary values problem:

$$
\left\{\begin{aligned}
-\Delta w & =0 & & \text { in } \Omega \backslash \bar{\omega} \\
w & =\partial_{\mathrm{n}} u-g & & \text { on } \partial \Omega \\
\partial_{\mathrm{n}} w+\alpha w+\beta \Delta_{\tau} w & =0 & & \text { on } \partial \omega .
\end{aligned}\right.
$$

Proof. We define, for $t \in[0, T)$,

$$
j(t)=J\left(\omega_{t}\right):=\frac{1}{2} \int_{\partial \Omega}\left|\partial_{\mathrm{n}} u_{t}-g\right|^{2},
$$

where $u_{t} \in \mathrm{H}^{1}\left(\Omega \backslash \overline{\omega_{t}}\right)$ solves Problem (5.1). Using Hadamard's formula, we immediately obtain

$$
\mathrm{D} J(\omega) \cdot \boldsymbol{V}=j^{\prime}(0)=\int_{\partial \Omega} \partial_{\mathrm{n}} u^{\prime}\left(\partial_{\mathrm{n}} u-g\right) .
$$

In order to obtain an expression of $j^{\prime}(0)$ as an integral on $\partial \omega$ with an explicit dependance in the perturbation direction $\boldsymbol{V}$, let us introduce the solution $w \in \mathrm{H}^{1}(\Omega \backslash \bar{\omega})$ of the adjoint problem (5.7). Hence, using $w$ as a test function in (5.2) and $u^{\prime}$ as a test function in (5.7), we use the Green's formula to obtain

$$
\int_{\partial(\Omega \backslash \bar{\omega})} \partial_{\mathrm{n}} u^{\prime} w=\int_{\partial(\Omega \backslash \bar{\omega})} \partial_{\mathrm{n}} w u^{\prime}
$$

Thus, adding $\int_{\partial \omega}\left(\alpha u^{\prime} w+\beta \Delta_{\tau} u^{\prime} w\right)$ in each side of the previous equality and using the fact that $\int_{\partial \omega} \beta \Delta_{\tau} u^{\prime} w=\int_{\partial \omega} \beta \Delta_{\tau} w u^{\prime}$,

$$
\int_{\partial \Omega} \partial_{\mathrm{n}} u^{\prime} w+\int_{\partial \omega}\left(\partial_{\mathrm{n}} u^{\prime}+\alpha u^{\prime}+\beta \Delta_{\tau} u^{\prime}\right) w=\int_{\partial \Omega} \partial_{\mathrm{n}} w u^{\prime}+\int_{\partial \omega}\left(\partial_{\mathrm{n}} w+\alpha w+\beta \Delta_{\tau} w\right) u^{\prime} .
$$

Using the boundary conditions satisfied by $u^{\prime}$ and $w$, we obtain the following characterization of the shape gradient of the functional

$$
\mathrm{D} J(\omega) \cdot \boldsymbol{V}=-\int_{\partial \omega} \xi\left(u, V_{\mathrm{n}}\right) w,
$$

where $\xi$ is given by (5.3). Finally, using integration by parts,

$$
\begin{aligned}
\mathrm{D} J(\omega) \cdot \boldsymbol{V}=-\int_{\partial \omega}\left[-\alpha \partial_{\mathrm{n}} u w\right. & -\alpha \mathrm{H} u w+\Delta_{\tau} u w-\operatorname{div}_{\tau}\left(\nabla_{\tau} u w\right) \\
& \left.-\beta \partial_{\mathrm{n}} u \Delta_{\tau} w+\beta\left(\mathrm{H} \nabla_{\tau} u \cdot \nabla_{\tau} w-2 \mathrm{D}^{2} b \nabla_{\tau} u \cdot \nabla_{\tau} w\right)\right] V_{\mathrm{n}} .
\end{aligned}
$$

Thus, since $-\beta \Delta_{\tau} w=\partial_{\mathrm{n}} w+\alpha w$ on $\partial \omega$, we obtain the announced result. 


\subsubsection{Second order shape derivatives of the functional}

Let us consider $\omega^{*} \in \mathcal{O}_{\delta}$ solution of the inverse problem (2.3). In order to study the stability of the optimization problem (2.6) at $\omega^{*}$, we want to compute the second order shape derivative of $J$, i.e. the shape Hessian. First of all, notice that we prove in exactly the same way than we proved the existence of the shape derivative $u^{\prime}$ that the adjoint state $w$ is differentiable with respect to the shape $\omega \in \Omega_{\delta}$ and we denote by $w^{\prime}$ its shape derivative. Then, we state the following result:

Proposition 5.3 (Characterization of the shape Hessian at a critical shape). For $\boldsymbol{V} \in \boldsymbol{U}$, we have

$\mathrm{D}^{2} J\left(\omega^{*}\right) \cdot \boldsymbol{V} \cdot \boldsymbol{V}=-\int_{\partial \omega^{*}}\left[-\alpha \mathrm{H} u w^{\prime}+\left(-\mathrm{I}+\beta\left(\mathrm{HI}-2 \mathrm{D}^{2} b\right)\left(\nabla_{\tau} u \cdot \nabla_{\tau} w^{\prime}\right)\right)+\partial_{\mathrm{n}} u \partial_{\mathrm{n}} w^{\prime}\right] V_{\mathrm{n}}$,

where $\mathrm{H}$ is the mean curvature of $\partial \omega^{*}, b$ is the signed distance and where $w^{\prime} \in \mathrm{H}^{1}\left(\Omega \backslash \overline{\omega^{*}}\right)$ is the solution of the following problem:

$$
\left\{\begin{aligned}
-\Delta w^{\prime} & =0 & & \text { in } \Omega \backslash \overline{\omega^{*}} \\
w^{\prime} & =\partial_{\mathrm{n}} u^{\prime} & & \text { on } \partial \Omega \\
\partial_{\mathrm{n}} w^{\prime}+\alpha w^{\prime}+\beta \Delta_{\tau} w^{\prime} & =0 & & \text { on } \partial \omega^{*} .
\end{aligned}\right.
$$

Proof. Using Hadamard's formula, we obtain

$$
j^{\prime \prime}(0)=\int_{\partial \Omega} \partial_{\mathrm{n}} u^{\prime \prime}\left(\partial_{\mathrm{n}} u-g\right)+\left|\partial_{\mathrm{n}} u^{\prime}\right|^{2} .
$$

We then characterize the shape derivative of the adjoint state in the same way that we characterized $u^{\prime}$, i.e.

$$
\left\{\begin{aligned}
-\Delta w^{\prime} & =0 & & \text { in } \Omega \backslash \bar{\omega} \\
w^{\prime} & =\partial_{\mathrm{n}} u^{\prime} & & \text { on } \partial \Omega \\
\partial_{\mathrm{n}} w^{\prime}+\alpha w^{\prime}+\beta \Delta_{\tau} w^{\prime} & =\xi\left(w, V_{\mathrm{n}}\right) & & \text { on } \partial \omega .
\end{aligned}\right.
$$

Noticing that for $\omega=\omega^{*}, \partial_{\mathrm{n}} u=g$ on $\partial \Omega$ and then $w \equiv 0$ in $\Omega \backslash \overline{\omega^{*}}$, we obtain the characterization (5.8). Then, using the same trick than in the computation of the first order shape derivative of the functional $J$ (with $u^{\prime}$ and $w^{\prime}$ as respective test functions in $(5.8)$ and $(5.2)$ ), we obtain

$$
\int_{\partial \Omega}\left|\partial_{\mathrm{n}} u^{\prime}\right|^{2}=\int_{\partial \omega}-\xi\left(u, V_{\mathrm{n}}\right) w^{\prime}
$$

We then conclude using the same manipulations than the ones used for the computations of the shape gradient (see proof of Proposition 5.2).

\subsection{Instability of the problem}

Now let us prove the instability of the inverse problem (2.3) using the method already used in [17, 6, 14, 13]. Thus, we use a local regularity argument in order to prove the compactness of the Riesz operator corresponding to the shape Hessian at a solution $\omega^{*} \in \mathcal{O}_{\delta}$ of the inverse problem. An alternative proof could be to use the potential layers as what is done in [2].

Proposition 5.4 (Compactness at a critical point). The Riesz operator corresponding to the shape Hessian $\mathrm{D}^{2} J\left(\omega^{*}\right)$ defined from $\mathbf{H}^{1 / 2}\left(\partial \omega^{*}\right)$ to $\mathbf{H}^{-1 / 2}\left(\partial \omega^{*}\right)$ is compact. 
Proof. The idea of the proof is to write the shape Hessian as a composition of linear continuous operators whose one is compact (the compactness being obtained using the compactness imbedding between two Sobolev spaces).

Let us focus on the term $\int_{\partial \omega^{*}} \alpha \mathrm{H} u w^{\prime} V_{\mathrm{n}}$ of the characterization of the shape Hessian given in Proposition 5.3. The other terms can be treated in the same way. We decompose this term as

$$
\int_{\partial \omega^{*}} \alpha \mathrm{H} u w^{\prime} V_{\mathrm{n}}=\langle K(\boldsymbol{V}), L(\boldsymbol{V})\rangle_{\mathrm{H}^{-1 / 2}\left(\partial \omega^{*}\right), \mathrm{H}^{1 / 2}\left(\partial \omega^{*}\right)},
$$

where

$$
\begin{aligned}
L & : \quad \boldsymbol{V} \in \mathbf{H}^{1 / 2}\left(\partial \omega^{*}\right) \mapsto \alpha \mathrm{H} u V_{\mathrm{n}} \in \mathrm{H}^{1 / 2}\left(\partial \omega^{*}\right) \\
K & : \quad \boldsymbol{V} \in \mathbf{H}^{1 / 2}\left(\partial \omega^{*}\right) \mapsto w^{\prime} \in \mathrm{H}^{-1 / 2}\left(\partial \omega^{*}\right) .
\end{aligned}
$$

Since $u$ is independent of $\boldsymbol{V}$, the operator $L$ is clearly linear continuous as multiplier by a smooth function (see [24]). Now, let us prove that the operator $K$ is compact which will be conclude the proof.

According to the characterization 5.8 of $w^{\prime}$, we decompose the operator $K$ as follows: $K=K_{2} \circ K_{1}$ with

$$
\begin{aligned}
& K_{1}: \quad \boldsymbol{V} \in \mathbf{H}^{1 / 2}\left(\partial \omega^{*}\right) \mapsto \partial_{\mathrm{n}} u^{\prime} \in \mathrm{H}^{1 / 2}(\partial \Omega) \\
& K_{2}: \Psi \in \mathrm{H}^{1 / 2}\left(\partial \omega^{*}\right) \mapsto \Phi \in \mathrm{H}^{-1 / 2}\left(\partial \omega^{*}\right),
\end{aligned}
$$

where $u^{\prime}$ is the solution of Problem $(5.2)$ and $\Phi \in \mathrm{H}^{1}\left(\Omega \backslash \overline{\omega^{*}}\right)$ solves

$$
\left\{\begin{aligned}
-\Delta \Phi & =0 & & \text { in } \Omega \backslash \overline{\omega^{*}} \\
\Phi & =\Psi & & \text { on } \partial \Omega \\
\partial_{\mathrm{n}} \Phi+\alpha \Phi+\beta \Delta_{\tau} \Phi & =0 & & \text { on } \partial \omega^{*} .
\end{aligned}\right.
$$

The operator $K_{1}$ is clearly linear continuous. Then, we decompose the operator $K_{2}$ as $K_{2}=K_{2,3} \circ K_{2,2} \circ K_{2,1}$ with

$$
\begin{aligned}
& K_{2,1}: \Psi \in \mathrm{H}^{1 / 2}(\partial \Omega) \mapsto \Phi \in \mathrm{H}^{3}\left(\Omega_{\delta} \backslash \overline{\omega^{*}}\right) \\
& K_{2,2}: \Phi \in \mathrm{H}^{3}\left(\Omega_{\delta} \backslash \overline{\omega^{*}}\right) \mapsto \Phi \in \mathrm{H}^{5 / 2}\left(\partial \omega^{*}\right) \\
& K_{2,3}: \Phi \in \mathrm{H}^{5 / 2}\left(\partial \omega^{*}\right) \mapsto \Phi \in \mathrm{H}^{-1 / 2}\left(\partial \omega^{*}\right) .
\end{aligned}
$$

Notice that the regularity $\mathrm{H}^{3}\left(\Omega_{\delta} \backslash \overline{\omega^{*}}\right)$ is due to a local regularity argument (as the one used in [6, 14, 13]): since the object $\omega^{*}$ has a $C^{3}$ boundary and since the condition on $\partial \omega^{*}$ is homogenous (and then smooth), the solution of Problem $(5.9)$ is globally $\mathrm{H}^{1}\left(\Omega \backslash \omega^{*}\right)$ but locally $\mathrm{H}^{3}\left(\Omega_{\delta} \backslash \overline{\omega^{*}}\right)$. The operators $K_{2,1}$ and $K_{2,2}$ are then linear continuous and the operator $K_{2,3}$ is the compact imbedding of $\mathrm{H}^{5 / 2}\left(\partial \omega^{*}\right)$ into $\mathrm{H}^{-1 / 2}\left(\partial \omega^{*}\right)$. Hence, we obtain the compactness result.

This result points out the lack of stability of the optimization problem (2.6): it means, roughly speaking, that, in a neighborhood of $\omega^{*}$ (i.e. for $t$ small), $J$ behaves as its second order approximation and one cannot expect an estimate of the kind $C t \leq \sqrt{J\left(\omega_{t}\right)}$ with a constant $C$ uniform in $\boldsymbol{V}$. Hence, this proposition emphasizes that the gradient has not a uniform sensitivity with respect to the deformation directions: $J$ is degenerate for highly oscillating perturbations. 


\section{$6 \quad$ Numerical experiments}

In this section, we want to make some numerical simulations in order to confirm and complete our previous theoretical results. We use a classical shape variation descent algorithm without any regularization method in order to solve numerically the optimization problem (2.6) as what is done in the cases of Dirichlet or Neumann boundary conditions (see for example [2, 14]). Indeed, it seems that, in our case, the degeneracy of the functional does not lead to apparition of oscillations in the numerical reconstruction. This problem seems to be less unstable than the cases of classical boundary conditions.

\subsection{Framework for the numerical simulations}

The numerical simulations presented are made in dimension two using the finite elements library FreEfem ++ (see [20]). We use a $P 2$ finite elements discretization to solve Problem (2.5) and the adjoint problem (5.7). The framework is the following: the exterior boundary is assumed to be the unit circle, the coefficients $\alpha, \beta$ are such that $\alpha=1$, $\beta=-0.1$ and we consider the exterior Dirichlet boundary condition

$$
f=\cos (\theta), \theta \in[0,2 \pi) .
$$

In order to have a suitable pair (measure $g$, domain $\omega^{*}$ ), we use a synthetic data: we fix a shape $\omega^{*}$, solve Problem 2.5 in $\Omega \backslash \overline{\omega^{*}}$ using another finite elements method (here a $P 3$ finite elements discretization) and extract the measurement $g$ by computing $\partial_{\mathrm{n}} u$ on $\partial \Omega$.

The optimization method used for the numerical simulations is here the classical gradient algorithm. We use initially $N_{\text {ext }}:=100$ discretization points for the exterior boundary and $N_{\text {int }}:=70$ points for the interior boundary. Then, we use the function movemesh of FrEEFEM + + in order to change the shape of the objects at each step and the function adaptmesh to refine and avoid degeneracy of the triangles in the meshes. In order to be completely explicit, we detail this algorithm:

\section{Algorithm 1}

1. fix a number of iterations $M$ and an initial shape $\omega_{0}$;

2. solve Problems (2.5) and (5.7) with $\omega=\omega_{i}$ (where $\omega_{i}$ denotes the $i^{\text {th }}$ iterate of the approximate shape);

3. compute the kernel of $\nabla J_{K V}\left(\omega_{i}\right)$ in Formula (5.6);

4. move the shape using the function movemesh;

5. get back to the step 2, while $i<M$.

Remark 6.1. We can notice that we have to solve only two problems (Problems (2.5) and (5.7) to compute the descent direction. Indeed, the directional perturbations $\boldsymbol{V}$ dependance is explicit in Formula 5.6.

The stopping criterion of the algorithm is here the number of iterations. Obviously, this criterion can be modified and even improved but this simple one permits to obtain efficient results. Moreover, we precise that we stop the algorithm if the value of the residual increase between two steps.

Let us be more precise about the step 4 of the algorithm. In order to have a descent direction, we solve the following problem

$$
\left\{\begin{aligned}
-\Delta \Phi+\Phi & =0 & & \text { in } \Omega \backslash \bar{\omega} \\
\partial_{\mathrm{n}} \Phi & =0 & & \text { on } \partial \Omega \\
\partial_{\mathrm{n}} \Phi & =-\left[-\alpha \mathrm{H} u w+\left(-\mathrm{I}+\beta\left(\mathrm{HI}-2 \mathrm{D}^{2} b\right)\right)\left(\nabla_{\tau} u \cdot \nabla_{\tau} w\right)+\partial_{\mathrm{n}} u \partial_{\mathrm{n}} w\right] & & \text { on } \partial \omega .
\end{aligned}\right.
$$


where the Neumann boundary condition on $\partial \omega$ is the kernel of the shape gradient $\nabla J$. Thus, the descent direction is given by $\nabla \Phi$. Let us precise that the step size is simply chosen testing the value of the functional: we decrease it if the value of $J$ increases and we increase it for the next iteration if the value of $J$ decreases.

\subsection{Reconstruction of some obstacles}

We first test our algorithm on simple shapes as circle or ellipse, centered or not. The results are quite efficient as showed in Figure 1. We precise here the color code that we choose
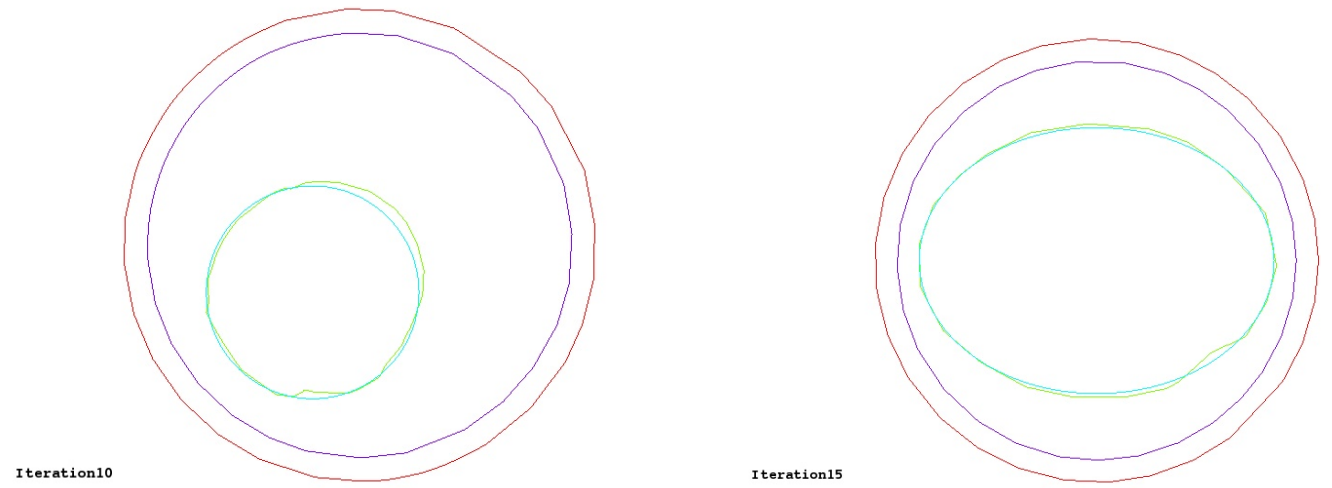

Figure 1: Reconstruction of simple shapes

and that we will use in the sequel. The exterior boundary is represented in red, the initial shape in purple, the exact shape in cyan and the obtained shape in green.

The reconstruction seems to be efficient to reconstruct "simple obstacles". Now, we aim to detect shapes with concavity or with corners. More precisely, we want to detect a square and a kite shape represented by

$$
\partial \omega_{1}:=\left\{\left(\begin{array}{c}
0.3(\cos \theta+0.65 \cos (2 \theta)-0.65) \\
0.45 \sin \theta
\end{array}\right), \theta \in[0,2 \pi)\right\} .
$$

We represent the results in Figure 2 where we see that the reconstruction is again efficient.
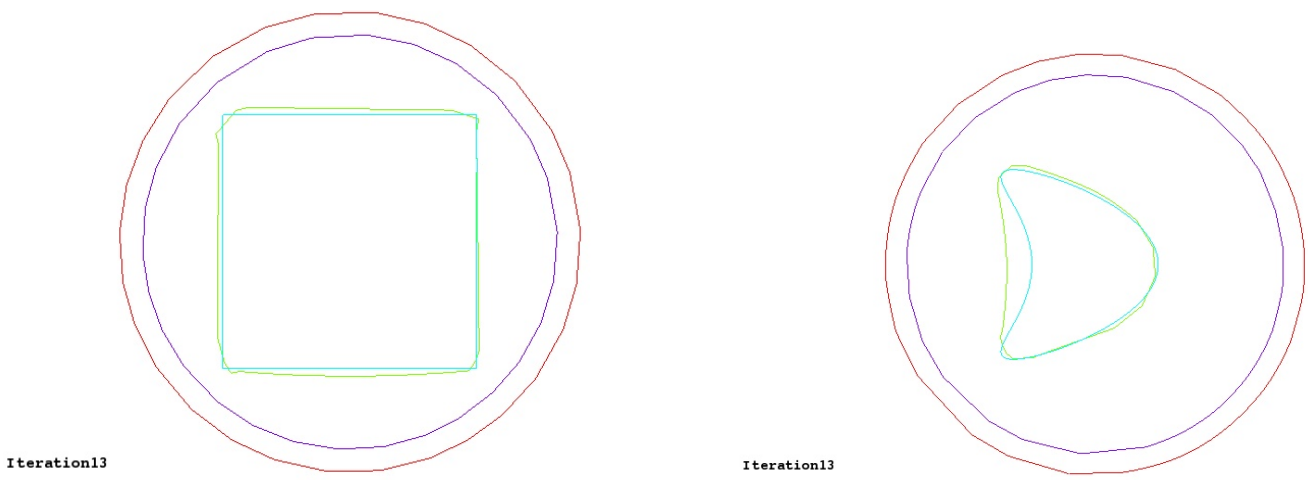

Figure 2: Reconstruction of non trivial shapes

Finally, we present in Figure 3 the reconstruction of the kite shape $\omega_{1}$ with respectively $3 \%$ and $10 \%$ artificial noise. Even if the error of detection increase with the percentage of noise, we obtain reasonable results. 

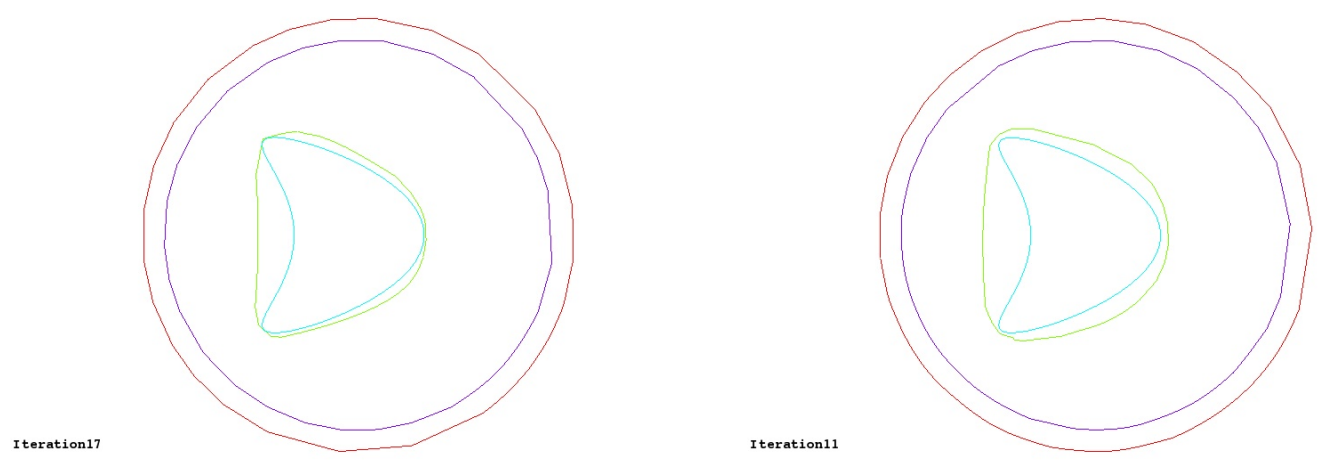

Figure 3: Reconstruction with $3 \%$ and $10 \%$ artificial noise

\subsection{Influence of some parameters}

Location and shape of the object. We wonder to know if our method is efficient to detect small obstacles. Thus, in Figure 4, we represent the result when we want to reconstruct a small square. We see that the detection is not good because of the object is then far away from the exterior boundary (that is where we make the measurement). It seems that, in this case, we detect efficiency the position of the obstacle but not its shape. We also see in Figure 4 that when we want to detect obstacles whose some parts are far
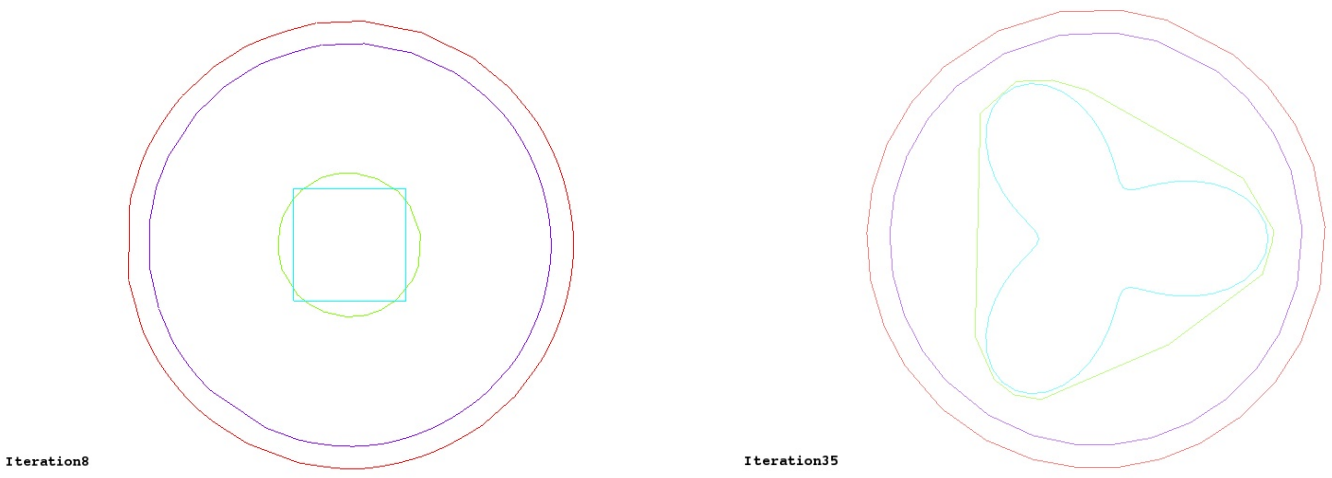

Figure 4: Detection non trivial shapes

away from the exterior boundary, the detection is not efficient. Here, the exact shape is represented by

$$
\partial \omega_{2}:=\left\{0.5(1+\cos (3 \theta))\left(\begin{array}{c}
\cos \theta \\
\sin \theta
\end{array}\right), \theta \in[0,2 \pi)\right\} .
$$

It seems that, in this case, we detect only the convex hull of the object.

Measurement. The imposed Dirichlet boundary conditions $f$ on $\partial \Omega$ seems to have an important role in the efficiency of the detection. Indeed, if we impose $f=\cos (5 \theta)$ instead of $f=\cos (\theta)(\theta \in[0,2 \pi))$, the result is significantly worse as represented in Figure 5 . where we want to reconstruct the kite shape $\omega_{1}$. 


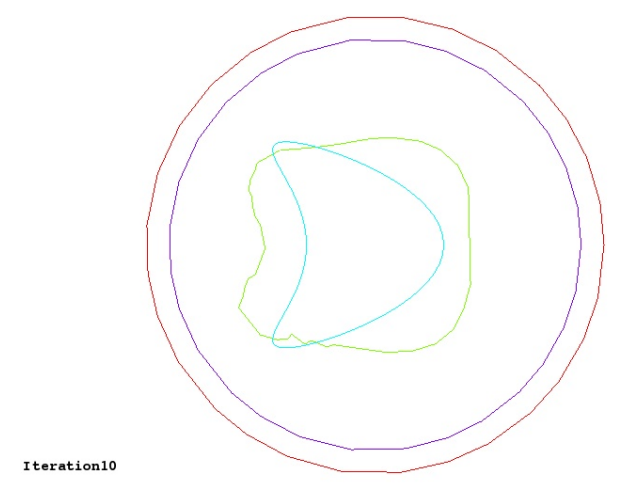

Figure 5: Detection non trivial shapes

\section{Conclusion}

We have partially reconstructed an obstacle immersed in a perfect fluid by minimizing a least squares functional using boundary measurements. The object $\omega$ was assumed to have a thin layer and was then modeled by using generalized boundary conditions (precisely Ventcel boundary conditions). We have first proved an identifiability result in the case of two concentric circles which claims that two pairs of measurements $\left(u_{i \mid \partial \Omega},\left.\partial_{\mathrm{n}} u_{i}\right|_{\partial \Omega}\right), i=1,2$ ensures the identification of the obstacle $\omega$. It is a first step in the investigations of the general identifiability result. Then, we have used shape optimization methods to compute the gradient of the cost functional and the shape Hessian. Thus, we have differentiated with respect to the domain the non classical Ventcel boundary conditions on $\partial \omega$. We have then proved that this problem is unstable in the sense that the functional degenerates for the highly oscillating perturbations of the boundary. However, the numerical simulations made in the bi-dimensional case were effective even if we do not use some regularization method to reconstruct the obstacle. It seems that the generalized impedance boundary conditions of order two lead to a problem less unstable than in the cases of classical boundary conditions.

This work may be followed by some others investigations. First of all, the general identifiability result for the shape from one or several boundary measurements is still an open problem. Moreover, a natural extension would be to study the determination of both the shape and the coefficients $\alpha, \beta$ of the Ventcel boundary conditions with the use of shape derivative.

\section{A Some results concerning Ventcel boundary conditions}

For reader's convenience, we here recall some results concerning the Poisson's equation with Ventcel boundary conditions. We refer to [29] and [7] for complements (see also [28]).

Let us first state a result concerning the Laplace-Beltrami operator (see for example [29] or 28] for details).

Theorem A.1. Let $s \in \mathbb{R}$ and $\omega$ a smooth open set. The operator

$$
-\Delta_{\tau}+\mathrm{I}: \mathrm{H}^{s+1}(\partial \omega) \rightarrow \mathrm{H}^{s-1}(\partial \omega)
$$

is an isomorphism.

Now let us prove the following existence and uniqueness result: 
Theorem A.2. Let $s \in \mathbb{R}$. Let $\Omega$ and $\omega$ be two smooth bounded connected open sets of $\mathbb{R}^{d}$ ( $d \geq 2$ and integer) such that $\omega \subset \subset \Omega$ and $\Omega \backslash \bar{\omega}$ is connected. Let $\alpha>0$ and $\beta<0$ two real numbers and let $f \in \mathrm{H}^{s+2}(\partial \Omega)$ and $g \in \mathrm{H}^{s}(\partial \omega)$. Then there exists a unique solution $u \in \mathrm{H}^{s+\frac{5}{2}}(\Omega \backslash \bar{\omega})$ of the following boundary value problem

$$
\left\{\begin{aligned}
-\Delta u & =0 & & \text { in } \Omega \\
u & =f & & \text { on } \partial \Omega \\
\partial_{\mathrm{n}} u+\alpha u+\beta \Delta_{\tau} u & =g & & \text { on } \partial \omega
\end{aligned}\right.
$$

and there exists a constant $C>0$ such that

$$
\|u\|_{\mathrm{H}^{s+\frac{5}{2}}(\Omega \backslash \bar{\omega})} \leq C\left(\|f\|_{\mathrm{H}^{s+2}(\partial \Omega)}+\|g\|_{\mathrm{H}^{s}(\partial \omega)}\right) .
$$

Proof. Let $F \in \mathrm{H}^{s+\frac{5}{2}}(\Omega)$ the unique solution of

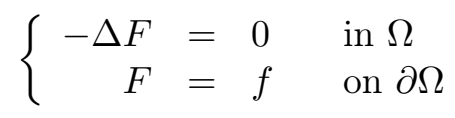

with $\|F\|_{\mathrm{H}^{s+\frac{5}{2}(\Omega)}} \leq C\|f\|_{\mathrm{H}^{s+2}(\partial \Omega)}$ (where $C>0$ is a constant). Then, Problem A.1 can be rewritten as the problem consisting of finding $\widetilde{u}:=u-F \in \mathrm{H}^{s+\frac{5}{2}}(\Omega)$ such that

$$
\left\{\begin{aligned}
-\Delta \widetilde{u} & =0 & & \text { in } \Omega \\
\widetilde{u} & =0 & & \text { on } \partial \Omega \\
\partial_{\mathrm{n}} \widetilde{u}+\alpha \widetilde{u}+\beta \Delta_{\tau} \widetilde{u} & =\widetilde{g} & & \text { on } \partial \omega,
\end{aligned}\right.
$$

where $\widetilde{g}:=g-\partial_{\mathrm{n}} F+\alpha F+\beta \Delta_{\tau} F$ on $\partial \omega$.

Now, let us introduce the Dirichlet-to-Neumann operator $\Lambda_{\omega}$ defined by

$$
\Lambda_{\omega}: U \in \mathrm{H}^{s+2}(\partial \omega) \mapsto \partial_{\mathrm{n}} \widetilde{u} \in \mathrm{H}^{s+1}(\partial \omega)
$$

where $\widetilde{u} \in \mathrm{H}^{s+\frac{5}{2}}(\Omega \backslash \bar{\omega})$ is the unique solution of

$$
\left\{\begin{aligned}
-\Delta \widetilde{u} & =0 & & \text { in } \Omega \\
\widetilde{u} & =0 & & \text { on } \partial \Omega \\
\widetilde{u} & =U & & \text { on } \partial \omega .
\end{aligned}\right.
$$

This operator $\Lambda_{\omega}$ is classically well-defined (see for example 23] for details). Notice that there exists a positive constant (still denoted by $C$ ) such that

$$
\|\widetilde{u}\|_{\mathrm{H}^{s+\frac{5}{2}}(\Omega \backslash \bar{\omega})} \leq C\|U\|_{\mathrm{H}^{s}(\partial \omega)} \cdot
$$

Then, Problem (A.2) (and thus Problem (A.1) can be rewritten as the surface equation

$$
\Lambda_{\omega} U+\alpha U+\beta \Delta_{\tau} U=\widetilde{g}
$$

where $U$ is the trace of $\widetilde{u}$ on $\partial \omega$. Problems $(\mathrm{A} .2$ and $(\mathrm{A} .3$ are clearly equivalent in the sense that if $\widetilde{u}$ solves A.2 , then its trace on $\partial \omega$ solves A.3 and, conversely, if $U$ solves (A.3), then its harmonic extension defined through $\Lambda_{\omega}$ solves A.2 .

Hence, we obtain the announced result if this surface equation A.3 admits a unique solution in $\mathrm{H}^{s+2}(\partial \omega)$ and if there exists a constant $C>0$ such that

$$
\|U\|_{\mathrm{H}^{s+2}(\partial \omega)} \leq C\|\widetilde{g}\|_{\mathrm{H}^{s}(\partial \omega)},
$$

which is proved in the following lemma. 
Lemma A.3. The surface equation A.3 admits a unique solution $U \in \mathrm{H}^{s+2}(\partial \omega)$ and there exists a constant $C>0$ such that

$$
\|U\|_{\mathrm{H}^{s+2}(\partial \omega)} \leq C\|\widetilde{g}\|_{\mathrm{H}^{s}(\partial \omega)} .
$$

Proof. Let us begin by considering the simplest situation $s=-1$. Then, the variational formulation of problem A.3 is given by

$$
\int_{\partial \omega} \Lambda_{\omega} U \phi+\alpha \int_{\partial \omega} U \phi-\beta \int_{\partial \omega} \nabla_{\tau} U \cdot \nabla_{\tau} \phi=\int_{\partial \omega} \widetilde{g} \phi \quad \forall \phi \in \mathrm{H}^{1}(\partial \omega) .
$$

Applying Lax-Milgram theorem to the bilinear form

$$
a(U, V):=\int_{\partial \omega} \Lambda_{\omega} U V+\alpha \int_{\partial \omega} U V-\beta \int_{\partial \omega} \nabla_{\tau} U \cdot \nabla_{\tau} V
$$

we prove the existence of a unique solution of Problem A.3 for $s=-1$ with the corresponding estimate. Indeed, the bilinear symmetric continuous form $a$ is coercive since

$$
\begin{aligned}
a(U, U) & =\int_{\partial \omega} \Lambda_{\omega} U U+\alpha\|U\|_{\mathrm{L}^{2}(\partial \omega)}^{2}-\beta\left\|\nabla_{\tau} U\right\|_{\mathrm{L}^{2}(\partial \omega)}^{2} \\
& \geq\|\nabla \widetilde{u}\|_{\mathrm{L}^{2}(\Omega \backslash \bar{\omega})}^{2}+\alpha\|U\|_{\mathrm{L}^{2}(\partial \omega)}^{2}-\beta\left\|\nabla_{\tau} U\right\|_{\mathrm{L}^{2}(\partial \omega)}^{2} \\
& \geq c\|\widetilde{u}\|_{\mathrm{H}^{1}(\Omega \backslash \bar{\omega})}^{2}+\alpha\|U\|_{\mathrm{L}^{2}(\partial \omega)}^{2}-\beta\left\|\nabla_{\tau} U\right\|_{\mathrm{L}^{2}(\partial \omega)}^{2} \\
& \geq c\|U\|_{\mathrm{L}^{2}(\partial \omega)}^{2}+\alpha\|U\|_{\mathrm{L}^{2}(\partial \omega)}^{2}-\beta\left\|\nabla_{\tau} U\right\|_{\mathrm{L}^{2}(\partial \omega)}^{2} \\
& \geq C\|u\|_{\mathrm{H}^{1}(\partial \omega)}^{2},
\end{aligned}
$$

where $C:=\min (c+\alpha,-\beta)>0$, since $\|U\|_{L^{2}(\partial \omega)}^{2}+\left\|\nabla_{\tau} U\right\|_{L^{2}(\partial \omega)}^{2}$ is (the square of) an equivalent norm in $\mathrm{H}^{1}(\partial \omega)$.

Consider now the case $s>-1$. Problem A.3 admits a unique solution $U \in \mathrm{H}^{1}(\partial \omega)$ using the previous step. Moreover

$$
-\Delta_{\tau} U+U=\frac{1}{-\beta}\left(\widetilde{g}-\Lambda_{\omega} U+(-\beta-\alpha) U\right) \in \mathrm{H}^{\min \{s, 0\}}(\partial \omega) .
$$

Hence, according to Theorem A.1 $U \in \mathrm{H}^{\min \{s+2,2\}}$. Consequently, by a bootstrap argument, we obtain $U \in \mathrm{H}^{s+2}(\partial \omega)$. The estimate is obtained using the Closed Graph Theorem since the operator

$$
\mathcal{V}:=\Lambda_{\omega}+\alpha \mathrm{I}+\beta \Delta_{\tau}: \mathrm{H}^{s+2}(\partial \omega) \rightarrow \mathrm{H}^{s}(\partial \omega)
$$

is bounded and since we just proved that it is bijective.

To conclude, let us treat the case $s<-1$ by transposition. The operator $\mathcal{V}$ continuously maps $\mathrm{H}^{s+2}(\partial \omega)$ into $\mathrm{H}^{s}(\partial \omega)$ for all $s \in \mathbb{R}$. Its adjoint is $\mathcal{V}$ himself which maps $\mathrm{H}^{-s}(\partial \omega)$ into $\mathrm{H}^{-s-2}(\partial \omega)$, which is bijective according to above since $-s-2>-1$. Hence, we conclude as in the previous case.

\section{B Shape differential calculus}

In this section, we recall some elements that are useful to compute shape derivatives. For more details on the shape calculus, the reader can consult the books [16] and 21] of Delfour et al. and of Henrot et al. In the sequel $\Omega$ will denote an open bounded set of $\mathbb{R}^{d}(d \geq 2)$ with a $C^{1}$ boundary. 


\section{B.1 Tangential differentiation}

Given $h \in C^{1}(\partial \Omega)$, we define the tangential gradient $\nabla_{\tau} h$ of $h$ by

$$
\nabla_{\tau} h:=\left.\left(\nabla \widetilde{h}-\partial_{\mathrm{n}} \widetilde{h} \mathbf{n}\right)\right|_{\partial \Omega} \quad \text { on } \partial \Omega,
$$

where $\widetilde{h} \in C^{1}\left(\mathbb{R}^{d}\right)$ is an extension of $h$. It is shown that the definition does not depend on the extension. Moreover, we extend this definition by density to the functions $h \in$ $\mathrm{W}^{1,1}(\partial \Omega)$. by

Given a vector field $\boldsymbol{W} \in \boldsymbol{C}^{1}(\partial \Omega)$, we define the tangential divergence $\operatorname{div}_{\tau} \boldsymbol{W}$ of $\boldsymbol{W}$

$$
\operatorname{div}_{\tau} \boldsymbol{W}:=\left.\left(\operatorname{div} \widetilde{\boldsymbol{W}}-\left(\left[\widetilde{\boldsymbol{W}}^{\prime}\right] \mathbf{n}\right) \cdot \mathbf{n}\right)\right|_{\partial \Omega} \quad \text { on } \partial \Omega,
$$

where $\widetilde{\boldsymbol{W}} \in \boldsymbol{C}^{1}\left(\mathbb{R}^{d}\right)$ is an extension of $\boldsymbol{W}$ and $\left[\widetilde{\boldsymbol{W}}^{\prime}\right]$ is the Jacobian matrix of $\widetilde{\boldsymbol{W}}$. It is shown that the definition does not depend on the extension. Moreover, we extend this definition by density to the functions $\boldsymbol{W} \in \mathbf{W}^{1,1}(\partial \Omega)$.

Assume that $\Omega$ has a $C^{2}$ boundary. We define the Laplacian-Beltrami operator $\Delta_{\tau} h$ of a function $h \in \mathrm{W}^{2,1}(\partial \Omega)$ by

$$
\Delta_{\tau} h:=\operatorname{div}_{\tau}\left(\nabla_{\tau} h\right) \quad \text { on } \partial \Omega .
$$

Let $u \in \mathrm{H}^{3}(\Omega)$. It is known (see for example [21, Proposition 5.4.12]) that the relation between $\Delta$ and $\Delta_{\tau}$ is given by

$$
\Delta u=\Delta_{\tau} u+\mathrm{H} \partial_{\mathrm{n}} u+\partial_{\mathrm{nn}}^{2} u \quad \text { on } \partial \Omega,
$$

where

$$
\partial_{\mathrm{nn}}^{2} u:=\left(\mathrm{D}^{2} u \mathbf{n}\right) \cdot \mathbf{n}=\sum_{i, j=1}^{d} \frac{\partial^{2} u}{\partial x_{i} \partial x_{j}} n_{i} n_{j}
$$

and where $\mathrm{H}$ is the mean curvature of $\partial \Omega$ defined by $\mathrm{H}:=\operatorname{div}_{\tau} \mathbf{n}$.

\section{B.2 Shape derivative of functionals}

We essentially need the result concerning the shape derivative of boundary integral that we recall here (see [21, Proposition 5.4.18]). Assume that $\Omega$ has a $C^{3}$ boundary. We consider the functional $J(\partial \Omega)=\int_{\partial \Omega} \Psi$, where $\Psi$ belongs to $\mathrm{W}^{2,1}(\partial \Omega)$. The shape derivative of $J$ in an admissible perturbation direction $\boldsymbol{V} \in \mathbf{W}^{1, \infty}\left(\mathbb{R}^{d}\right)$ is given by

$$
\mathrm{d} J(\partial \Omega, \boldsymbol{V})=\int_{\partial \Omega} \Psi^{\prime}+\int_{\partial \Omega}\left(\partial_{\mathrm{n}} \Psi+\mathrm{H} \Psi\right)(\boldsymbol{V} \cdot \mathbf{n}),
$$

where $\Psi^{\prime}$ is the shape derivative of $\Psi$.

\section{B.3 Shape tangential derivation}

When $\partial \Omega$ is subject to a deformation, we have to define an extension of the exterior unit normal $\mathbf{n}$ to a no variable domain. This can be done with the signed distance to $\partial \Omega$. Then, we recall an important result that we need to compute shape derivative of the least squares functional. 
For $x \in \mathbb{R}^{d}$, we define $b(x, \partial \Omega)$ the signed distance to $\partial \Omega$ by

$$
b(x, \partial \Omega):=\left\{\begin{array}{rll}
\mathrm{d}(x, \partial \Omega) & \text { if } & x \in \mathbb{R}^{d} \backslash \bar{\Omega} \\
0 & \text { if } & x \in \partial \Omega \\
-\mathrm{d}(x, \partial \Omega) & \text { if } & x \in \Omega .
\end{array}\right.
$$

In [21, page 198-199], it is shown that $\nabla b(x, \partial \Omega)$ is a smooth extension of the exterior unit normal $\mathbf{n}$. Furthermore, we have

$$
\mathbf{n}=\left.\nabla b(x, \partial \Omega)\right|_{\partial \Omega} \quad \text { on } \partial \Omega
$$

and $\mathrm{D}^{2} b=\nabla n=\nabla_{\tau} n$ is symmetric in a tubular neighborhood of $\mathbb{R}^{d}$. We can resume the needed result on $b$ in the following two results.

Proposition B.1. The shape derivative of $\mathbf{n}$ is

$$
\mathbf{n}^{\prime}=-\nabla_{\tau}(\boldsymbol{V} \cdot \mathbf{n}),
$$

where $\boldsymbol{V}$ represents a perturbed direction.

Proof. Since $\nabla b \cdot \nabla b=1$, we have $\left(|\nabla b|^{2}\right)^{\prime}=0$, i.e. $\nabla b^{\prime} \cdot \nabla b=0$. Hence

$$
\left.\nabla b^{\prime}\right|_{\partial \Omega}=\left.\nabla_{\tau} b^{\prime}\right|_{\partial \Omega}+\left(\nabla b^{\prime} \cdot \mathbf{n}\right) \mathbf{n}=\left.\nabla_{\tau} b^{\prime}\right|_{\partial \Omega} .
$$

Since $b^{\prime}=-\boldsymbol{V} \cdot \mathbf{n}$ on $\partial \Omega$, we have $\mathbf{n}^{\prime}=\left.\nabla_{\tau} b^{\prime}\right|_{\partial \Omega}=-\left.\nabla_{\tau}(\boldsymbol{V} \cdot \mathbf{n})\right|_{\partial \Omega}$.

Theorem B.2. Given $u, \varphi \in C^{2}(\bar{\Omega})$, we have

$$
\partial_{\mathrm{n}}\left(\nabla_{\tau} u \cdot \nabla_{\tau} \varphi\right)=\nabla_{\tau}\left(\partial_{\mathrm{n}} u\right) \cdot \nabla_{\tau} \varphi+\nabla_{\tau} \varphi \cdot \nabla_{\tau}\left(\partial_{\mathrm{n}} u\right)-2 \mathrm{D}^{2} b \nabla_{\tau} u \cdot \nabla_{\tau} \varphi,
$$

where $b$ is the signed distance to $\partial \Omega$.

Proof. We have, on $\partial \Omega$,

$$
\begin{aligned}
\partial_{\mathrm{n}}\left(\nabla_{\tau} u \cdot \nabla_{\tau} \varphi\right)= & \nabla\left(\nabla_{\tau} u \cdot \nabla_{\tau} \varphi\right) \cdot \mathbf{n} \\
= & \nabla\left(\nabla u \cdot \nabla \varphi-\partial_{\mathrm{n}} u \partial_{\mathrm{n}} \varphi\right) \cdot \mathbf{n} \\
= & \mathrm{D}^{2} u \nabla \varphi \cdot \mathbf{n}+\mathrm{D}^{2} \varphi \nabla u \cdot \mathbf{n}-\partial_{\mathrm{nn}}^{2} u \partial_{\mathrm{n}} \varphi-\partial_{\mathrm{nn}}^{2} \varphi \partial_{\mathrm{n}} u \\
= & \partial_{\mathrm{n}} \varphi\left(\mathrm{D}^{2} u \mathbf{n}\right) \cdot \mathbf{n}+\left(\mathrm{D}^{2} u \nabla_{\tau} \varphi\right) \cdot \mathbf{n}+\partial_{\mathrm{n}} u \mathrm{D}^{2} \varphi \mathbf{n} \cdot \mathbf{n} \\
& \quad+\mathrm{D}^{2} \varphi \nabla_{\tau} u \cdot \mathbf{n}-\partial_{\mathrm{nn}}^{2} u \partial_{\mathrm{n}} \varphi-\partial_{\mathrm{nn}}^{2} \varphi \partial_{\mathrm{n}} u \\
= & \left(\mathrm{D}^{2} u \nabla_{\tau} \varphi\right) \cdot \mathbf{n}+\left(\mathrm{D}^{2} \varphi \nabla_{\tau} u\right) \cdot \mathbf{n} .
\end{aligned}
$$

Moreover, since $\nabla \mathbf{n}=\mathrm{D}^{2} b$, we have

$$
\nabla(\nabla u \cdot \mathbf{n}) \cdot \nabla_{\tau} \varphi=\left(\mathrm{D}^{2} u \mathbf{n}\right) \cdot \nabla_{\tau} \varphi+\left(\mathrm{D}^{2} b \nabla u\right) \cdot \nabla_{\tau} \varphi
$$

and since $\mathrm{D}^{2} b \mathbf{n}=0$, it comes

$$
\nabla(\nabla u \cdot \mathbf{n}) \cdot \nabla_{\tau} \varphi=\left(\mathrm{D}^{2} u \mathbf{n}\right) \cdot \nabla_{\tau} \varphi+\left(\mathrm{D}^{2} b \nabla_{\tau} u\right) \cdot \nabla_{\tau} \varphi .
$$

Hence,

$$
\left(\mathrm{D}^{2} u \nabla_{\tau} \varphi\right) \cdot \mathbf{n}=\nabla_{\tau}\left(\partial_{\mathbf{n}} u\right) \cdot \nabla_{\tau} \varphi-\mathrm{D}^{2} b \nabla_{\tau} u \cdot \nabla_{\tau} \varphi .
$$

Reporting in (B.1), we get the announced result (since $(\overline{B .2})$ is also valid interchanging the role of $u$ and $\varphi$ ). 


\section{References}

[1] R. A. Adams and J. J. F. Fournier. Sobolev spaces, volume 140 of Pure and Applied Mathematics (Amsterdam). Elsevier/Academic Press, Amsterdam, second edition, 2003.

[2] L. Afraites, M. Dambrine, K. Eppler, and D. Kateb. Detecting perfectly insulated obstacles by shape optimization techniques of order two. Discrete Contin. Dyn. Syst. Ser. B, 8(2):389-416 (electronic), 2007.

[3] L. Afraites, M. Dambrine, and D. Kateb. On second order shape optimization methods for electrical impedance tomography. SIAM J. Control Optim., 47(3):1556-1590, 2008.

[4] X. Antoine and H. Barucq. Approximation by generalized impedance boundary conditions of a transmission problem in acoustic scattering. M2AN Math. Model. Numer. Anal., 39(5):1041-1059, 2005.

[5] V. Bacchelli. Uniqueness for the determination of unknown boundary and impedance with the homogeneous Robin condition. Inverse Problems, 25(1):015004, 4, 2009.

[6] M. Badra, F. Caubet, and M. Dambrine. Detecting an obstacle immersed in a fluid by shape optimization methods. Math. Models Methods Appl. Sci., 21(10):2069-2101, 2011.

[7] V. Bonnaillie-Noël, M. Dambrine, F. Hérau, and G. Vial. On generalized Ventcel's type boundary conditions for Laplace operator in a bounded domain. SIAM J. Math. Anal., 42(2):931-945, 2010.

[8] L. Bourgeois, N. Chaulet, and H. Haddar. Stable reconstruction of generalized impedance boundary conditions. Inverse Problems, 27(9):095002, 26, 2011.

[9] L. Bourgeois and H. Haddar. Identification of generalized impedance boundary conditions in inverse scattering problems. Inverse Probl. Imaging, 4(1):19-38, 2010.

[10] F. Cakoni and R. Kress. Integral equations for inverse problems in corrosion detection from partial Cauchy data. Inverse Probl. Imaging, 1(2):229-245, 2007.

[11] F. Cakoni and R. Kress. Integral equations methods for the inverse obstacle problems with generalized impedance boundary condition. Inverse Problems, 29:015005, 2012.

[12] F. Caubet. Detecting an obstacle immersed in a fluid: the Stokes case. Jaca: Proceedings, 35:91-101, 2010.

[13] F. Caubet. Instability of an inverse problem for the stationary Navier-Stokes equations. HAL-00696174, 2012. (submitted).

[14] F. Caubet, M. Dambrine, D. Kateb, and C. Z. Timimoun. A Kohn-Vogelius formulation to detect an obstacle immersed in a fluid. HAL-00678036, 2012. (submitted).

[15] M. Dambrine and D. Kateb. Persistency of wellposedness of Ventcel's boundary value problem under shape deformations. J. Math. Anal. Appl., 394(1):129-138, 2012.

[16] M. C. Delfour and J.-P. Zolésio. Shapes and geometries, volume 22 of Advances in Design and Control. Society for Industrial and Applied Mathematics (SIAM), Philadelphia, PA, second edition, 2011. Metrics, analysis, differential calculus, and optimization. 
[17] K. Eppler and H. Harbrecht. A regularized Newton method in electrical impedance tomography using shape Hessian information. Control Cybernet., 34(1):203-225, 2005.

[18] H. Haddar, P. Joly, and H.-M. Nguyen. Generalized impedance boundary conditions for scattering by strongly absorbing obstacles: the scalar case. Math. Models Methods Appl. Sci., 15(8):1273-1300, 2005.

[19] H. Haddar, P. Joly, and H.-M. Nguyen. Generalized impedance boundary conditions for scattering problems from strongly absorbing obstacles: the case of Maxwell's equations. Math. Models Methods Appl. Sci., 18(10):1787-1827, 2008.

[20] F. Hecht. Finite Element Library FreEFEM ++ . http://www.freefem.org/ff++/.

[21] A. Henrot and M. Pierre. Variation et optimisation de formes, volume 48 of Mathématiques \& Applications (Berlin) [Mathematics \& Applications]. Springer, Berlin, 2005. Une analyse géométrique. [A geometric analysis].

[22] R. Kress and W. Rundell. Nonlinear integral equations and the iterative solution for an inverse boundary value problem. Inverse Problems, 21(4):1207-1223, 2005.

[23] J.-L. Lions and E. Magenes. Problèmes aux limites non homogènes et applications. Vol. 1. Travaux et Recherches Mathématiques, No. 17. Dunod, Paris, 1968.

[24] V. Maz'ya and T. Shaposhnikova. Theory of multipliers in spaces of differentiable functions, volume 23 of Monographs and Studies in Mathematics. Pitman (Advanced Publishing Program), Boston, MA, 1985.

[25] F. Murat and J. Simon. Sur le contrôle par un domaine géométrique. Rapport du L.A. 189, 1976. Université de Paris VI, France.

[26] C. Poignard. Generalized impedance boundary condition at high frequency for a domain with thin layer: the circular case. Appl. Anal., 86(12):1549-1568, 2007.

[27] J. Sokołowski and J.-P. Zolésio. Introduction to shape optimization, volume 16 of Springer Series in Computational Mathematics. Springer-Verlag, Berlin, 1992. Shape sensitivity analysis.

[28] M. E. Taylor. Partial differential equations, volume 23 of Texts in Applied Mathematics. Springer-Verlag, New York, 1996. Basic theory.

[29] J. L. Vázquez and E. Vitillaro. On the Laplace equation with dynamical boundary conditions of reactive-diffusive type. J. Math. Anal. Appl., 354(2):674-688, 2009. 